\title{
Temporal dynamics of amino and fatty acid composition in the razor clam Ensis siliqua (Mollusca: Bivalvia)
}

\author{
Miguel Baptista • Tiago Repolho • Ana Luísa Maulvault • \\ Vanessa M. Lopes · Luis Narciso • António Marques • \\ Narcisa Bandarra $\cdot$ Rui Rosa
}

Received: 21 November 2013/Revised: 10 April 2014/ Accepted: 11 June 2014/Published online: 22 June 2014

(C) Springer-Verlag Berlin Heidelberg and AWI 2014

\begin{abstract}
Few studies have been conducted on the temporal dynamics of both amino acid (AA) and fatty acid (FA) profiles in marine bivalves. We investigated the seasonal variation of these compounds in the pod razor clam Ensis siliqua in relation to food availability, salinity, water temperature and reproductive cycle. AA content varied between 46.94 and $54.67 \%$ dry weight (DW), and the AAs found in greater quantity were glutamic acid, glycine and aspartic acid. FA content varied between 34.02 and $87.94 \mathrm{mg} \mathrm{g}^{-1} \mathrm{DW}$ and the FAs found in greater quantity were 16:0 and 22:6n-3. Seasonal trends were observed for AAs and FAs. FAs increased with
\end{abstract}

Miguel Baptista and Tiago Repolho have contributed equally to this work.

Communicated by H.-D. Franke.

M. Baptista ( $\square)$ · T. Repolho · V. M. Lopes · L. Narciso ·

R. Rosa

Laboratório Marítimo da Guia, Centro de Oceanografia,

Faculdade de Ciências, Universidade de Lisboa, Av. Nossa

Senhora do Cabo, 939, 2750-374 Cascais, Portugal

e-mail: miguelnogueirabaptista@gmail.com

T. Repolho

e-mail: tfrepolho@fc.ul.pt

L. Narciso

e-mail: 1fnarciso@fc.ul.pt

R. Rosa

e-mail: rarosa@fc.ul.pt

A. L. Maulvault · A. Marques - N. Bandarra

Divisão de Aquacultura e Valorização, IPMA, Avenida de

Brasília, 1449-006 Lisbon, Portugal

e-mail: scubana@hotmail.com

A. Marques

e-mail: amarques@ipma.pt gametogenesis and decreased with spawning while AA content increased throughout spawning. The effect of increasing temperature and high food availability during the spawning season masked the loss of AAs resulting from gamete release. Still, a comparatively greater increase in the contents of glutamic acid and leucine with spawning indicate their possible involvement in a post-spawning gonad recovery mechanism. A post-spawning decrease in $14: 0,16: 0,16: 1 n-7,18: 1 n-7$ and 18:1n-9 is indicative of the importance of these FAs in bivalve eggs. An increase in 18:3n-3, 18:4n-3, 20:1n-9 and 20:2n-6 during gametogenesis suggests their involvement in oocyte maturation. The FA 22:4n-6, while increasing with spawning, appears to play a role in post-spawning gonad recovery. Salinity did not have an effect on the AA composition. None of the environmental parameters measured had an effect on FA composition.

Keywords Amino acids - Fatty acids - Biochemical profile $\cdot$ Seasonality $\cdot$ Razor clam $\cdot$ Ensis siliqua

\section{Introduction}

Bivalves are primary consumers that constitute an important link in the aquatic food chain (Hamdani and SoltaniMazouni 2011). Their significant ecological role and economical relevance have long contributed to a generous amount of academic focus. As a result, information on the biochemistry of a wide range of bivalve species became available (e.g., Bayne et al. 1978; Lewin et al. 1979; Jarzebski et al. 1986; Wenne and Styczyńska-Jurewicz 1987 and references therein). Studies of seasonal changes in biochemical content of organisms allow understanding how environment, ecology and physiology can affect their 
composition. As such, seasonal changes in the biochemistry of bivalves are known to be linked to the complex interaction of both biotic factors (e.g., growth, reproduction and food availability) and abiotic factors (e.g., temperature and salinity) (Gabbott 1983). Levels of proteins, lipids and carbohydrates (glycogen) have been shown to fluctuate with food availability and reproductive cycle. Food abundance allows for the accumulation of proteins and lipids in bivalve tissues (Taylor and Venn 1979; Bressan and Marin 1985; Wenne and Styczyńska-Jurewicz 1987). Lipids generally increase during the course of gametogenesis (Barber and Blake 1981; Tarnowska et al. 2009; Yang et al. 2011) and decrease upon release of gametes (i.e., spawning; Pieters et al. 1980; Bressan and Marin 1985; Tarnowska et al. 2009). For proteins, diverging trends have been observed throughout gametogenesis and spawning. During gametogenesis, protein content was found to increase (Joaquim et al. 2008; Tarnowska et al. 2009; Yang et al. 2011), decrease (Taylor and Venn 1979; Davis and Wilson 1983) and even remain stable (Pieters et al. 1980). During spawning, levels of protein were found to increase (Davis and Wilson 1983; Yang et al. 2011) and decrease (Pieters et al. 1980; Bressan and Marin 1985). Differences in food availability and water temperature conditions may partially explain the observed discrepancies since these factors are known to influence protein accumulation (Taylor and Venn 1979). Focusing on proteins and lipids, compounds involved in most biochemical and physiological processes of any organism is therefore useful for the recognition of ecological (e.g., Bressan and Marin 1985; Darriba et al. 2005a) and physiological changes (e.g., Beukema and De Bruin 1977; Davis and Wilson 1983; Tarnowska et al. 2009). Greater detail in the measurement of biochemical changes is achieved, however, when analyzing amino acids (AAs) and fatty acids (FAs). Indeed, differences in seasonal trend have been observed among both AAs and FAs (e.g., Beninger and Stephan 1985; Fatima 1996; Ojea et al. 2004). More commonly reported, is the different behavior exhibited among free AAs in relation to salinity (Sokolowski et al. 2003) and that exhibited among FAs in relation to temperature (Holland 1978). Studies conducted so far provided a general view of the seasonal variations in bivalve biochemistry, but few assess the temporal variations occurring among AAs and FAs. Most studies focusing on AAs in bivalves investigate the effects of salinity change on the levels of free AAs (Allen 1961; Ellis 1985; Sokolowski et al. 2003; Kube et al. 2007) or the AA composition of shells (Dussart 1983; Goodfriend et al. 1997; Barbour Wood et al. 2006) or specific soft body parts (i.e., gills and mantle; Trytek and Allen 1980). Few studies report whole-body AA composition of bivalves (Sidwell et al. 1979; Fatima 1996; Özden and Erkan 2011). Whole-body FA composition has received greater attention (e.g., Lewin et al. 1979; Beninger and Stephan 1985; Teshima et al. 1990; Pazos et al. 1996; Taylor and Savage 2006). Still, among these studies, only four evaluated seasonality: one on the AA composition (Fatima 1996) and three on the FA composition (Beninger and Stephan 1985; Pazos et al. 1996; Taylor and Savage 2006).

The pod razor clam Ensis siliqua (Linnaeus 1758) is found over a wide latitudinal range, from the Norwegian Sea along the Eastern Atlantic and west coast of Africa, and also the Baltic and Mediterranean seas (Tebble 1966; von Cosel and Gofas 2011). It inhabits fine sand, silt or sandy-mud bottoms of coastal zones where they can form extensive beds commonly found between 3 and $7 \mathrm{~m}$ although occurring until $12 \mathrm{~m}$ (Tebble 1966; Monteiro and Gaspar 1993; Gaspar et al. 1999). They form deep burrows (Hauton et al. 2003; Wootton et al. 2003) with longitudinal axis of the shell in a vertical orientation (da Costa et al. 2010) and when feeding both siphons protrude above the sediment (Tuck et al. 2000; Breen et al. 2011). This species has a single spawning period (Lebour 1938; Gaspar and Monteiro 1998; Darriba et al. 2005b) with males and females exhibiting synchronism in the gametogenic development and spawning (Gaspar and Monteiro 1998). Studies on coastal waters of the Iberian Peninsula show that gametogenesis starts in November with spawning occurring mainly during April and May (Gaspar and Monteiro 1998; Darriba et al. 2005b). The pod razor clam is a high-value commercial species that has been heavily fished along the Portuguese coast since 1969 (Gaspar and Monteiro 1998; Gaspar et al. 1999). Though attention has been paid to the population genetics of this species (Fernández-Tajes et al. 2007, 2012) and limited studies conducted on its gametogenic cycle (Gaspar and Monteiro 1998; Darriba et al. 2005b), the AA and FA composition of this razor clam has not been assessed.

Thus, this study aims to evaluate the seasonal variation in AA and FA composition of $E$. siliqua over a 1-year period in relation to food availability, salinity, water temperature and reproductive cycle. FA levels are expected to increase with greater food availability and throughout gametogenesis and decrease with spawning. AA levels are expected to increase with food availability while no prediction is possible on reproduction-related fluctuations. Salinity and water temperature may also affect AA and FA composition.

\section{Materials and methods}

Biological sampling

Ensis siliqua individuals were hand collected in Caldeira de Tróia, a shallow water habitat near the mouth of Sado 
estuary, Portugal. Sampling was performed at low tide, in the intertidal zone, once every 2 months, from April 2010 to February 2011.

For the biochemical analysis, three pooled samples $(n=20)$ were taken from 60 individuals selected for shell size $(\geq 60 \mathrm{~mm})$ indicative of reproductive capability (Gaspar and Monteiro 1998). The soft tissue was removed and then homogenized with a grinder (Retsch Grindomix GM200, Düsseldorf, Germany; 5,000 rpm; material: PP cup and stainless steel knifes), vacuum packed and frozen at $-80{ }^{\circ} \mathrm{C}$. The frozen samples were freeze-dried for $48 \mathrm{~h}$ at $-50{ }^{\circ} \mathrm{C}$ under low pressure (approximately $10^{-1}$ atm), powdered and stored at $-80{ }^{\circ} \mathrm{C}$. Moisture content was determined according to the AOAC (2005) methodologies, by drying the sample overnight at $105^{\circ} \mathrm{C}$ (laboratory heater, P-Selecta 207).

\section{Amino acid analysis}

To extract total amino acids (protein bound + free), $14.8-15.8 \mathrm{mg}$ of sample were placed in 10-ml ampoules with $3 \mathrm{ml}$ of $6 \mathrm{M} \mathrm{HCl}$ (Merck) containing $0.1 \%$ phenol (Merck), according to the method described by AOAC (2005). Following the establishment of inert and anaerobic conditions, to prevent oxidative degradation of amino acids, ampules were sealed and samples hydrolyzed at $110{ }^{\circ} \mathrm{C}$ for $24 \mathrm{~h}$; hydrolysates were filtered $(0.45 \mu \mathrm{m}$ pore size) and dissolved with Milli-Q distilled water to $20 \mathrm{ml}$. Samples were then frozen at $-80{ }^{\circ} \mathrm{C}$ and freeze-dried for $48 \mathrm{~h}$ at $-50{ }^{\circ} \mathrm{C}$ under low pressure (approximately $\left.10^{-1} \mathrm{~atm}\right)$, after which they were dissolved in $5 \mathrm{ml}$ of $0.1 \mathrm{M} \mathrm{HCl}$ (Merck) and stored at $-80{ }^{\circ} \mathrm{C}$ until amino acid separation. Finally, thawed samples were filtered $(0.2 \mu \mathrm{m}$ pore size) and separation was performed with high-performance liquid chromatography (Agilent 1100 HPLC) using precolumn o-phthalaldehyde and 3-mercaptopropionic acid in borate buffer (OPA, Agilent Technologies) and 9-fluorenylmethylchloroformate in acetonitrile (FMOC; Agilent Technologies) derivatization, a Phenomenex Gemini ODS C18 guard column $(4 \mathrm{~mm} \times 3 \mathrm{~mm})$ and a Phenomenex Gemini ODS C18 110A column $(4.6 \mathrm{~mm} \times 150 \mathrm{~mm}, 5 \mu \mathrm{m})$. The solvents and gradient conditions were those described by Henderson et al. (2000). Detection wavelengths were set at UV 338 and $262 \mathrm{~nm}$ and fluorescence $340 / 450$ and 266/305 nm. The identity and quantity of the amino acids were assessed by comparison with the retention times and peak areas of standard amino acids (Sigma-Aldrich) using norvaline and sarcosine as internal standards. Trypthophan and cysteine were quantified in E. siliqua; however, because these are partially lost during the acidic hydrolysis, they were not considered for analysis.
Fatty acid analysis

Fatty acid methyl esters (FAME) profile from nonpolar and polar lipids was determined in triplicate for each sample and was based on the experimental procedure of Cohen et al. (1988); 300-330 mg of sample were dissolved in $5 \mathrm{~mL}$ of acetyl chloride/methanol (1:19 v/v; Merck), shaken and heated $\left(80^{\circ} \mathrm{C} ; 1 \mathrm{~h}\right)$. After cooling, $1 \mathrm{~mL}$ of Milli$\mathrm{Q}$ distilled water and $2 \mathrm{~mL}$ of $n$-heptane pro analysis (Merck) were added, and samples were shaken and centrifuged $(2,300 \mathrm{~g}, 5 \mathrm{~min}$; 3K30 Sigma Laborzentrifugen GmbH, Osterode am Harz, Germany) until phase separation. The moisture content of the upper phase was removed using anhydrous sodium sulfate (Panreac). A $2 \mu \mathrm{L}$ aliquot from the upper phase was then injected onto a gas chromatograph (Varian Star 3800 Cp, Walnut Creek, CA, USA) equipped with an autosampler and fitted with a flame ionization detector at $250{ }^{\circ} \mathrm{C}$ for FAME analysis. Fatty acid separation was carried out with helium as carrier gas at

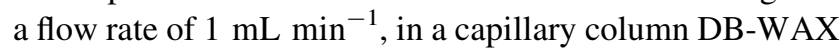
(30 m length $\times 0.32 \mathrm{~mm}$ internal diameter; $0.25 \mu \mathrm{m}$ film thickness; Hewlett-Packard, Albertville, MN) programmed at $180{ }^{\circ} \mathrm{C}$ for $5 \mathrm{~min}$, raised to 220 at $4{ }^{\circ} \mathrm{C} \mathrm{min}{ }^{-1}$, and maintained at $220^{\circ} \mathrm{C}$ for $25 \mathrm{~min}$, with the injector at $250{ }^{\circ} \mathrm{C}$. FAME identification was accomplished through comparison of retention times with those of Sigma standards. Quantitative data were obtained with Varian software using C21:0 FA (Sigma) as internal standard.

\section{Environmental parameters}

To ascertain the existence of a relationship between AA and FA levels in E. siliqua and environmental conditions in Caldeira de Tróia, environmental data were obtained from the Annual Reports of Tróia Monitoring Program in IMAR (2008-2011). Data on turbidity (here as proxy of food availability for these suspension feeders), salinity and water temperature acquired between September 2007 and September 2010 (during high tide) were used. The compiled data set was not continuous for the shellfish sampling period. Consequently, data were pooled into four seasonal periods: Spring (1 March-31 May), Summer (1 June-31 August), Autumn (1 September-30 November) and Winter (1 December-28 February).

Statistical analysis

Seasonal differences in the AA and FA composition and environmental conditions were tested with analysis of variance (ANOVA) followed by multiple comparisons test (Tukey's test). Where necessary, data were transformed $\left(\log _{10}\right)$ to correct for homogeneity of variance. When data transformation still did not meet the assumptions of 
ANOVA, differences were analyzed with the nonparametric ANOVA equivalent (Kruskall-Wallis). Pearson's correlation coefficients were used to identify the relationships among the potential explanatory environmental variables for biochemical composition patterns (Table 1). Simple and multiple regression analyses were used to test for associations among variables. The combined effect of the three environmental parameters (turbidity, salinity and water temperature) yielded akin or, mostly, lower $R^{2}$ values than those obtained with the more explanatory pair of parameters for each AA and FA. All statistical analyses were tested at 0.05 level of probability with the software

Table 1 Pearson's correlation coefficients between turbidity, salinity and water temperature in Caldeira de Tróia between September 2007 and September 2010

\begin{tabular}{lll}
\hline & Turbidity & Salinity \\
\hline Turbidity & 1 & \\
Salinity & -0.37 & 1 \\
Water temperature & -0.26 & 0.10 \\
\hline
\end{tabular}

Correlation coefficients are non-significant $(P>0.05)$
STATISTICATM 6.1 (Statsoft, Inc., Tulsa, OK 74104, USA).

\section{Results}

Seasonal variations in amino acid composition

The general trend among AAs exhibited two peaks (June and February) and two minimums (April and December). A few different trends were, however, observed (Table 2). The main essential amino acids (EAAs) were arginine (Fig. 1C), leucine and lysine. The content of leucine was greater in June when compared to April and December (Fig. 1B, Tukey's test, $F=6.11$, df $=5, P<0.01$ ). Methionine values did not vary significantly throughout the year (Fig. 1A, Tukey's test, $F=2.18$, df $=5, P>0.05$ ). Total EAA ( $\left.\sum E A A\right)$ content was significantly greater in June and August when compared to April and December (Fig. 1D, Tukey's test, $F=7.27$, df $=5, P<0.01$ ).

The main non-essential amino acids (NEAAs) were glutamic acid, glycine (Fig. 2B) and aspartic acid.

Table 2 Amino acid composition (\% dry weight) of Ensis siliqua, between April 2010 and February 2011

\begin{tabular}{|c|c|c|c|c|c|c|}
\hline \multirow[t]{2}{*}{ Amino acids } & \multirow{2}{*}{$\begin{array}{l}\text { Spring } \\
\text { April }\end{array}$} & \multicolumn{2}{|l|}{ Summer } & \multirow{2}{*}{$\begin{array}{l}\text { Autumn } \\
\text { October }\end{array}$} & \multicolumn{2}{|l|}{ Winter } \\
\hline & & June & August & & December & February \\
\hline \multicolumn{7}{|l|}{ Essential (EAA) } \\
\hline Threonine & $2.54 \pm 0.03^{\mathrm{a}}$ & $2.83 \pm 0.19^{\mathrm{a}}$ & $2.79 \pm 0.05^{\mathrm{a}}$ & $2.66 \pm 0.08^{\mathrm{a}}$ & $2.64 \pm 0.05^{\mathrm{a}}$ & $2.84 \pm 0.10^{\mathrm{a}}$ \\
\hline Valine & $2.15 \pm 0.02^{\mathrm{a}}$ & $2.38 \pm 0.08^{\mathrm{b}}$ & $2.30 \pm 0.05^{\mathrm{ab}}$ & $2.21 \pm 0.11^{\mathrm{ab}}$ & $2.13 \pm 0.02^{\mathrm{a}}$ & $2.36 \pm 0.05^{\mathrm{b}}$ \\
\hline Methionine & $0.90 \pm 0.02^{\mathrm{a}}$ & $0.97 \pm 0.19^{\mathrm{a}}$ & $1.10 \pm 0.06^{\mathrm{a}}$ & $1.13 \pm 0.15^{\mathrm{a}}$ & $0.89 \pm 0.07^{\mathrm{a}}$ & $1.04 \pm 0.05^{\mathrm{a}}$ \\
\hline Isoleucine & $1.94 \pm 0.02^{\mathrm{a}}$ & $2.20 \pm 0.09^{\mathrm{b}}$ & $2.17 \pm 0.05^{\mathrm{bc}}$ & $2.08 \pm 0.09^{\mathrm{abc}}$ & $1.99 \pm 0.03^{\mathrm{ac}}$ & $2.20 \pm 0.07^{\mathrm{b}}$ \\
\hline Leucine & $3.49 \pm 0.03^{\mathrm{a}}$ & $4.16 \pm 0.22^{\mathrm{b}}$ & $4.08 \pm 0.17^{\mathrm{bc}}$ & $3.87 \pm 0.24^{\mathrm{abc}}$ & $3.64 \pm 0.04^{\mathrm{ac}}$ & $4.05 \pm 0.18^{\mathrm{bc}}$ \\
\hline Phenylalanine & $1.85 \pm 0.02^{\mathrm{a}}$ & $2.06 \pm 0.08^{\mathrm{bc}}$ & $2.05 \pm 0.05^{\mathrm{abc}}$ & $1.90 \pm 0.11^{\mathrm{abc}}$ & $1.86 \pm 0.01^{\mathrm{ab}}$ & $2.10 \pm 0.07^{\mathrm{c}}$ \\
\hline Histidine & $0.96 \pm 0.01^{\mathrm{abc}}$ & $1.02 \pm 0.03^{\mathrm{bc}}$ & $1.00 \pm 0.04^{\mathrm{abc}}$ & $0.93 \pm 0.04^{\mathrm{ab}}$ & $0.92 \pm 0.02^{\mathrm{a}}$ & $1.04 \pm 0.03^{\mathrm{c}}$ \\
\hline Lysine & $3.64 \pm 0.19^{\mathrm{ab}}$ & $4.21 \pm 0.08^{\mathrm{a}}$ & $3.83 \pm 0.36^{\mathrm{ab}}$ & $3.59 \pm 0.10^{\mathrm{b}}$ & $3.46 \pm 0.06^{\mathrm{b}}$ & $3.98 \pm 0.26^{\mathrm{ab}}$ \\
\hline Arginine & $3.71 \pm 0.03^{\mathrm{ab}}$ & $4.25 \pm 0.05^{\mathrm{ab}}$ & $4.54 \pm 0.13^{\mathrm{a}}$ & $4.01 \pm 0.32^{\mathrm{ab}}$ & $3.20 \pm 0.04^{\mathrm{b}}$ & $4.09 \pm 0.56^{\mathrm{ab}}$ \\
\hline$\sum \mathrm{EAA}$ & $21.19 \pm 0.24^{\mathrm{ab}}$ & $24.08 \pm 0.68^{\mathrm{c}}$ & $23.86 \pm 1.04^{\mathrm{c}}$ & $22.39 \pm 1.28^{\mathrm{abc}}$ & $20.73 \pm 0.21^{\mathrm{a}}$ & $23.70 \pm 1.38^{\mathrm{bc}}$ \\
\hline \multicolumn{7}{|c|}{ Non-essential (NEAA) } \\
\hline Aspartic acid & $4.78 \pm 0.06^{\mathrm{a}}$ & $5.58 \pm 0.29^{\mathrm{ab}}$ & $5.78 \pm 0.16^{\mathrm{b}}$ & $5.34 \pm 0.32^{\mathrm{ab}}$ & $4.96 \pm 0.03^{\mathrm{ab}}$ & $5.56 \pm 0.27^{\mathrm{ab}}$ \\
\hline Tyrosine & $1.70 \pm 0.01^{\mathrm{a}}$ & $1.94 \pm 0.08^{\mathrm{b}}$ & $1.94 \pm 0.06^{\mathrm{b}}$ & $1.82 \pm 0.11^{\mathrm{ab}}$ & $1.73 \pm 0.01^{\mathrm{a}}$ & $1.96 \pm 0.05^{\mathrm{b}}$ \\
\hline Serine & $2.41 \pm 0.01^{\mathrm{ab}}$ & $2.59 \pm 0.05^{\mathrm{a}}$ & $2.50 \pm 0.09^{\mathrm{ab}}$ & $2.35 \pm 0.15^{\mathrm{ab}}$ & $2.31 \pm 0.01^{\mathrm{b}}$ & $2.50 \pm 0.08^{\mathrm{ab}}$ \\
\hline Glutamic acid & $7.12 \pm 0.09^{\mathrm{a}}$ & $8.58 \pm 0.43^{b c}$ & $8.67 \pm 0.30^{\mathrm{c}}$ & $8.17 \pm 0.48^{\mathrm{abc}}$ & $7.46 \pm 0.09^{\mathrm{ab}}$ & $8.21 \pm 0.54^{\mathrm{abc}}$ \\
\hline Glycine & $5.17 \pm 0.10^{\mathrm{ab}}$ & $5.87 \pm 0.30^{\mathrm{bc}}$ & $6.11 \pm 0.22^{\mathrm{c}}$ & $5.83 \pm 0.38^{\mathrm{bc}}$ & $4.86 \pm 0.03^{\mathrm{bd}}$ & $4.23 \pm 0.19^{\mathrm{d}}$ \\
\hline Alanine & $3.71 \pm 0.06^{\mathrm{ab}}$ & $4.01 \pm 0.17^{\mathrm{b}}$ & $3.57 \pm 0.11^{\mathrm{a}}$ & $3.42 \pm 0.13^{\mathrm{ac}}$ & $3.05 \pm 0.04^{\mathrm{c}}$ & $3.34 \pm 0.23^{\mathrm{ac}}$ \\
\hline Proline & $1.82 \pm 0.04^{\mathrm{ab}}$ & $2.02 \pm 0.07^{\mathrm{ab}}$ & $1.98 \pm 0.07^{\mathrm{ab}}$ & $1.77 \pm 0.14^{\mathrm{a}}$ & $1.83 \pm 0.05^{\mathrm{ab}}$ & $2.04 \pm 0.14^{\mathrm{b}}$ \\
\hline$\sum$ NEAA & $26.72 \pm 0.31^{\mathrm{a}}$ & $30.59 \pm 1.26^{\mathrm{b}}$ & $30.56 \pm 1.00^{\mathrm{b}}$ & $28.73 \pm 1.83^{\mathrm{ab}}$ & $26.21 \pm 0.12^{\mathrm{a}}$ & $27.84 \pm 1.63^{\mathrm{ab}}$ \\
\hline$\sum \mathrm{AA}$ & $47.91 \pm 0.18^{\mathrm{a}}$ & $54.67 \pm 1.83^{\mathrm{b}}$ & $54.42 \pm 2.04^{\mathrm{b}}$ & $51.11 \pm 3.08^{\mathrm{ab}}$ & $46.94 \pm 0.31^{\mathrm{a}}$ & $51.53 \pm 2.99^{\mathrm{ab}}$ \\
\hline
\end{tabular}

Different superscript letters within rows represent significant differences $(P<0.05)$ and should be interpreted in the following exemplificative manner: $\mathbf{a}$ not $\neq \mathbf{a}, \mathbf{a} \neq \mathbf{b}, \mathbf{a} \neq \mathbf{c} ; \mathbf{a b} \neq \mathbf{c d}, \mathbf{a}$ not $\neq \mathbf{a b}, \mathbf{a b}$ not $\neq \mathbf{b c}$

Values are means of triplicate samples (+SD). Amino acid essentiality was defined based on Knauer and Southgate (1999) 

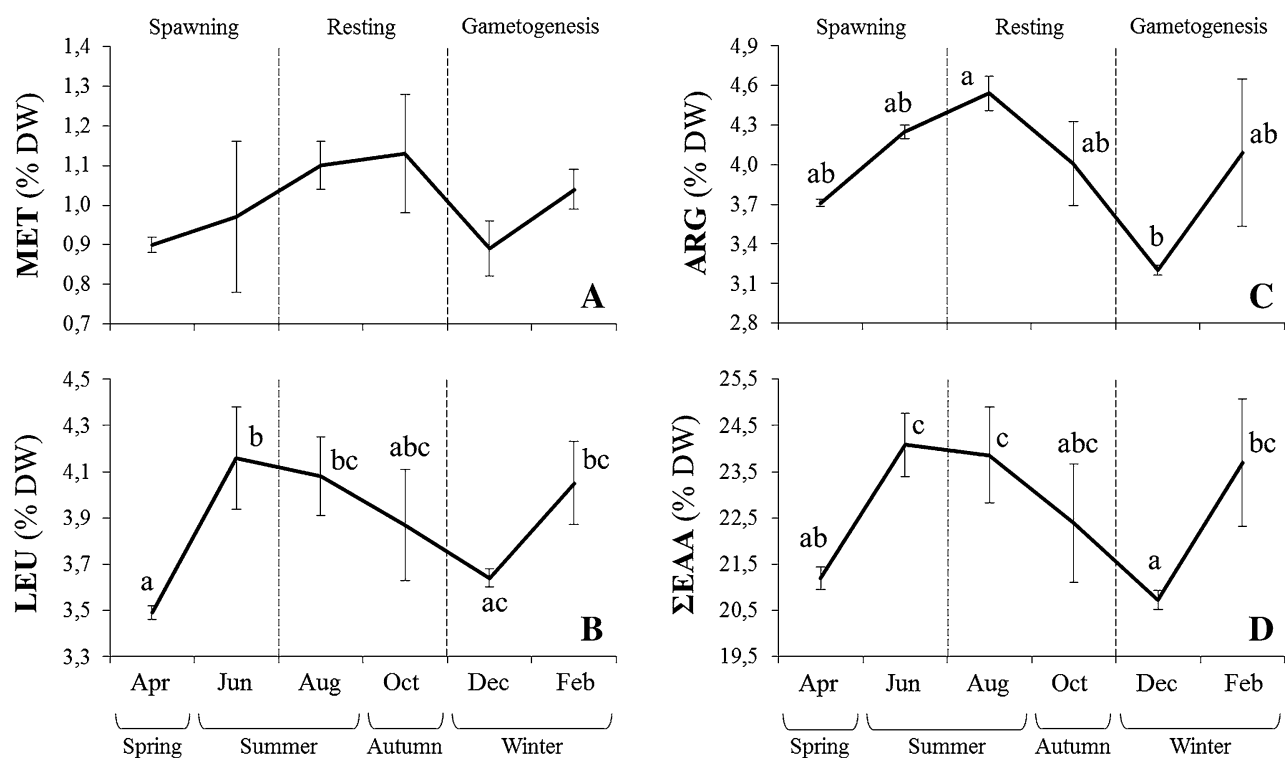

Fig. 1 Main essential amino acids content and total essential amino acid content, between April 2010 and February 2011. A Methionine, $\mathbf{B}$ leucine, $\mathbf{C}$ arginine, $\mathbf{D}$ total essential amino acid content. Values are means of triplicate samples $( \pm \mathrm{SD})$. Different superscript letters represent significant differences between months $(P<0.05)$ and
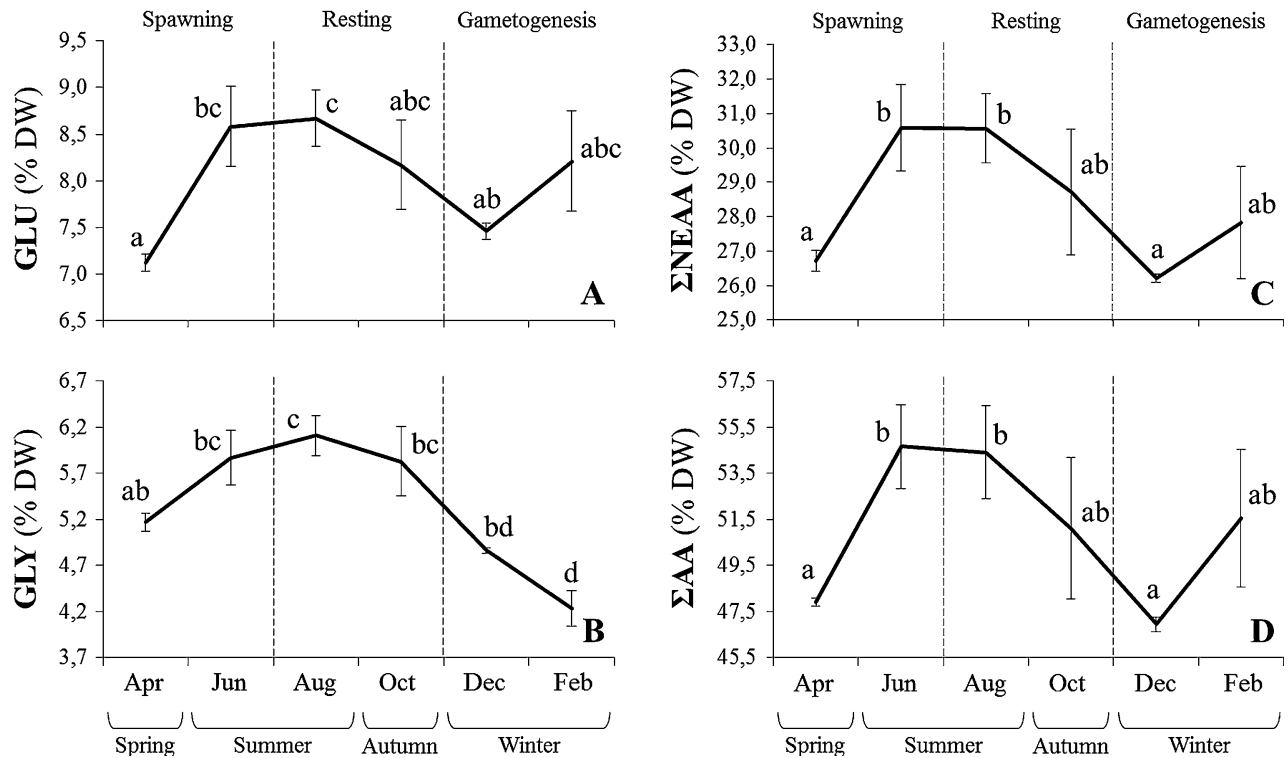

Fig. 2 Main non-essential amino acids content, total non-essential amino acid content and total amino acid content, between April 2010 and February 2011. A Glutamic acid, B glycine, $\mathbf{C}$ total non-essential amino acid content, $\mathbf{D}$ total amino acid content. Values are means of triplicate samples $( \pm \mathrm{SD})$. Different superscript letters represent

Glutamic acid content was greater in August in relation to April and December (Fig. 2A, Tukey's test, $F=6.91$, $\mathrm{df}=5, P<0.01)$. Differently, values for proline were higher in February than in October (Table 2, Tukey's test, $F=4.74$, df $=5, P<0.05$ ). Similarly to $\sum E A A$, total

should be interpreted in the following exemplificative manner: $\mathbf{a}$ not $\neq \mathbf{a}, \mathbf{a} \neq \mathbf{b}, \mathbf{a} \neq \mathbf{c} ; \mathbf{a b} \neq \mathbf{c d}, \mathbf{a}$ not $\neq \mathbf{a b}, \mathbf{a b}$ not $\neq \mathbf{b c}$. Temporal evolution in gonad development stages as determined by Gaspar and Monteiro (1998) is presented. MET methionine, LEU leucine, $A R G$ arginine, $\Sigma E A A$ total essential amino acid content

significant differences between months $(P<0.05)$ and should be interpreted as exemplified in Fig. 1 caption. Temporal evolution in gonad development stages as determined by Gaspar and Monteiro (1998) is presented. GLU glutamic acid, GLY glycine, $\Sigma N E A A$ total non-essential amino acid content, $\Sigma A A$ total amino acid content

NEAA ( $\sum$ NEAA) content was significantly greater in June and August when compared to April and December (Fig. 2C, Tukey's test, $F=7.24$, df $=5, \quad P<0.01$ ). Concomitantly, total amino acid ( $\left.\sum \mathrm{AA}\right)$ content was also significantly greater in June and August when compared to 
Table 3 Fatty acid composition (mg g ${ }^{-1}$ dry weight) of Ensis siliqua between April 2010 and February 2011

\begin{tabular}{|c|c|c|c|c|c|c|}
\hline \multirow[t]{2}{*}{ Fatty acids } & \multirow{2}{*}{$\begin{array}{l}\text { Spring } \\
\text { April }\end{array}$} & \multicolumn{2}{|l|}{ Summer } & \multirow{2}{*}{$\begin{array}{l}\text { Autumn } \\
\text { October }\end{array}$} & \multicolumn{2}{|l|}{ Winter } \\
\hline & & June & August & & December & February \\
\hline \multicolumn{7}{|l|}{ Saturated (SFA) } \\
\hline $14: 0$ & $4.57 \pm 1.48^{\mathrm{a}}$ & $2.65 \pm 0.05^{\mathrm{b}}$ & $0.83 \pm 0.15^{\mathrm{c}}$ & $0.72 \pm 0.26^{\mathrm{c}}$ & $0.78 \pm 0.02^{\mathrm{c}}$ & $0.79 \pm 0.08^{\mathrm{c}}$ \\
\hline $15: 0$ & $0.41 \pm 0.11^{\mathrm{a}}$ & $0.30 \pm 0.01^{\mathrm{ab}}$ & $0.16 \pm 0.03^{\mathrm{c}}$ & $0.16 \pm 0.05^{\mathrm{c}}$ & $0.19 \pm 0.00^{\mathrm{bc}}$ & $0.22 \pm 0.02^{\mathrm{bc}}$ \\
\hline $16: 0$ & $19.01 \pm 4.16^{\mathrm{a}}$ & $11.17 \pm 0.73^{\mathrm{b}}$ & $5.31 \pm 0.79^{c}$ & $4.84 \pm 1.34^{\mathrm{c}}$ & $4.99 \pm 0.12^{\mathrm{c}}$ & $5.43 \pm 0.55^{\mathrm{c}}$ \\
\hline $17: 0$ & $0.88 \pm 0.14^{\mathrm{a}}$ & $0.77 \pm 0.07^{\mathrm{ab}}$ & $0.51 \pm 0.08^{\mathrm{c}}$ & $0.45 \pm 0.12^{\mathrm{c}}$ & $0.50 \pm 0.01^{\mathrm{c}}$ & $0.58 \pm 0.05^{\mathrm{bc}}$ \\
\hline $18: 0$ & $6.04 \pm 0.66^{\mathrm{a}}$ & $5.27 \pm 0.23^{\mathrm{a}}$ & $3.48 \pm 0.39^{\mathrm{b}}$ & $2.95 \pm 0.69^{\mathrm{b}}$ & $2.87 \pm 0.05^{\mathrm{b}}$ & $3.16 \pm 0.19^{\mathrm{b}}$ \\
\hline 19:0 & $0.20 \pm 0.03^{\mathrm{a}}$ & $0.19 \pm 0.01^{\mathrm{a}}$ & $0.12 \pm 0.02^{\mathrm{b}}$ & $0.10 \pm 0.03^{\mathrm{b}}$ & $0.10 \pm 0.00^{\mathrm{b}}$ & $0.11 \pm 0.00^{\mathrm{b}}$ \\
\hline $20: 0$ & $0.43 \pm 0.27^{\mathrm{a}}$ & $0.56 \pm 0.11^{\mathrm{a}}$ & $0.39 \pm 0.18^{\mathrm{a}}$ & $0.34 \pm 0.11^{\mathrm{a}}$ & $0.35 \pm 0.01^{\mathrm{a}}$ & $0.38 \pm 0.05^{\mathrm{a}}$ \\
\hline $22: 0$ & $0.12 \pm 0.00^{\mathrm{ab}}$ & $0.16 \pm 0.05^{\mathrm{a}}$ & $0.00 \pm 0.00^{\mathrm{b}}$ & $0.05 \pm 0.00^{\mathrm{ab}}$ & $0.07 \pm 0.01^{\mathrm{ab}}$ & $0.07 \pm 0.01^{\mathrm{ab}}$ \\
\hline \multicolumn{7}{|l|}{ Branched } \\
\hline Iso $15: 0$ & $0.07 \pm 0.00^{\mathrm{a}}$ & $0.03 \pm 0.00^{\mathrm{a}}$ & $0.02 \pm 0.01^{\mathrm{a}}$ & $0.02 \pm 0.01^{\mathrm{a}}$ & $0.02 \pm 0.00^{\mathrm{a}}$ & $0.03 \pm 0.00^{\mathrm{a}}$ \\
\hline Anteiso 16:0 & $0.53 \pm 0.08^{\mathrm{a}}$ & $0.42 \pm 0.05^{\mathrm{a}}$ & $0.43 \pm 0.08^{\mathrm{a}}$ & $0.39 \pm 0.11^{\mathrm{a}}$ & $0.44 \pm 0.01^{\mathrm{a}}$ & $0.56 \pm 0.04^{\mathrm{a}}$ \\
\hline Iso $17: 0$ & $0.80 \pm 0.15^{\mathrm{a}}$ & $0.55 \pm 0.04^{\mathrm{b}}$ & $0.37 \pm 0.07^{\mathrm{b}}$ & $0.33 \pm 0.09^{\mathrm{b}}$ & $0.36 \pm 0.01^{\mathrm{b}}$ & $0.49 \pm 0.04^{\mathrm{b}}$ \\
\hline$\sum$ Branched & $1.38 \pm 0.28^{\mathrm{a}}$ & $1.00 \pm 0.03^{\mathrm{ab}}$ & $0.82 \pm 0.16^{\mathrm{b}}$ & $0.73 \pm 0.20^{\mathrm{b}}$ & $0.81 \pm 0.02^{\mathrm{b}}$ & $1.07 \pm 0.07^{\mathrm{ab}}$ \\
\hline$\sum \mathrm{SFA}$ & $33.04 \pm 7.13^{\mathrm{a}}$ & $22.07 \pm 1.22^{\mathrm{b}}$ & $11.61 \pm 1.78^{\mathrm{c}}$ & $10.35 \pm 2.79^{\mathrm{c}}$ & $10.64 \pm 0.22^{\mathrm{c}}$ & $11.81 \pm 1.02^{\mathrm{c}}$ \\
\hline \multicolumn{7}{|c|}{ Monounsaturated (MUFA) } \\
\hline $16: 1 n-9$ & $0.84 \pm 0.07^{\mathrm{ab}}$ & $0.72 \pm 0.08^{\mathrm{a}}$ & $0.73 \pm 0.11^{\mathrm{a}}$ & $0.64 \pm 0.16^{\mathrm{a}}$ & $0.69 \pm 0.01^{\mathrm{a}}$ & $1.06 \pm 0.06^{\mathrm{b}}$ \\
\hline $16: 1 n-7$ & $3.73 \pm 0.72^{\mathrm{a}}$ & $2.82 \pm 0.12^{\mathrm{b}}$ & $0.87 \pm 0.13^{\mathrm{c}}$ & $0.76 \pm 0.23^{\mathrm{c}}$ & $0.65 \pm 0.08^{\mathrm{c}}$ & $0.78 \pm 0.08^{\mathrm{c}}$ \\
\hline $16: 1 n-5$ & $0.16 \pm 0.03^{\mathrm{a}}$ & $0.12 \pm 0.01^{\mathrm{ab}}$ & $0.08 \pm 0.01^{b c}$ & $0.06 \pm 0.01^{\mathrm{c}}$ & $0.06 \pm 0.00^{\mathrm{c}}$ & $0.08 \pm 0.01^{\mathrm{bc}}$ \\
\hline $17: 1$ & $0.96 \pm 0.15^{\mathrm{a}}$ & $0.81 \pm 0.16^{\mathrm{ab}}$ & $0.76 \pm 0.13^{\mathrm{ab}}$ & $0.50 \pm 0.11^{\mathrm{b}}$ & $0.70 \pm 0.01^{\mathrm{ab}}$ & $0.88 \pm 0.05^{\mathrm{a}}$ \\
\hline $18: 1 n-9$ & $5.12 \pm 0.99^{\mathrm{a}}$ & $2.81 \pm 0.06^{\mathrm{b}}$ & $1.50 \pm 0.09^{c}$ & $1.30 \pm 0.35^{\mathrm{c}}$ & $1.20 \pm 0.04^{\mathrm{c}}$ & $1.24 \pm 0.06^{\mathrm{c}}$ \\
\hline $18: 1 n-7$ & $2.36 \pm 0.42^{\mathrm{a}}$ & $1.51 \pm 0.02^{\mathrm{b}}$ & $0.90 \pm 0.11^{\mathrm{c}}$ & $0.84 \pm 0.22^{\mathrm{c}}$ & $1.03 \pm 0.05^{\mathrm{bc}}$ & $1.13 \pm 0.04^{\mathrm{bc}}$ \\
\hline $18: 1 n-5$ & $0.00 \pm 0.00^{\mathrm{a}}$ & $0.03 \pm 0.00^{\mathrm{a}}$ & $0.00 \pm 0.00^{\mathrm{a}}$ & $0.00 \pm 0.00^{\mathrm{a}}$ & $0.02 \pm 0.00^{\mathrm{a}}$ & $0.00 \pm 0.00^{\mathrm{a}}$ \\
\hline $20: 1 n-11$ & $1.17 \pm 0.11^{\mathrm{a}}$ & $1.25 \pm 0.04^{\mathrm{a}}$ & $1.13 \pm 0.16^{\mathrm{ab}}$ & $0.83 \pm 0.21^{\mathrm{bc}}$ & $0.77 \pm 0.02^{\mathrm{c}}$ & $0.81 \pm 0.01^{\mathrm{c}}$ \\
\hline $20: 1 n-9$ & $2.12 \pm 0.21^{\mathrm{a}}$ & $1.19 \pm 0.06^{\mathrm{b}}$ & $0.80 \pm 0.11^{\mathrm{c}}$ & $0.68 \pm 0.17^{\mathrm{c}}$ & $0.67 \pm 0.02^{\mathrm{c}}$ & $0.68 \pm 0.02^{\mathrm{c}}$ \\
\hline $20: 1 n-7$ & $0.84 \pm 0.08^{\mathrm{a}}$ & $0.76 \pm 0.02^{\mathrm{ab}}$ & $0.57 \pm 0.09^{\mathrm{ab}}$ & $0.46 \pm 0.11^{\mathrm{b}}$ & $0.57 \pm 0.01^{\mathrm{ab}}$ & $0.77 \pm 0.01^{\mathrm{ab}}$ \\
\hline $22: 1 n-9$ & $0.00 \pm 0.00^{\mathrm{a}}$ & $0.00 \pm 0.00^{\mathrm{a}}$ & $0.00 \pm 0.00^{\mathrm{a}}$ & $0.03 \pm 0.00^{\mathrm{a}}$ & $0.00 \pm 0.00^{\mathrm{a}}$ & $0.06 \pm 0.00^{\mathrm{a}}$ \\
\hline$\sum$ MUFA & $17.29 \pm 2.79^{\mathrm{a}}$ & $12.01 \pm 0.28^{\mathrm{b}}$ & $7.35 \pm 0.84^{\mathrm{c}}$ & $6.08 \pm 1.59^{\mathrm{c}}$ & $6.32 \pm 0.23^{c}$ & $7.46 \pm 0.33^{\mathrm{c}}$ \\
\hline \multicolumn{7}{|c|}{ Polyunsaturated (PUFA) } \\
\hline $16: 2 n-4$ & $0.24 \pm 0.01^{\mathrm{a}}$ & $0.27 \pm 0.02^{\mathrm{a}}$ & $0.25 \pm 0.04^{\mathrm{a}}$ & $0.25 \pm 0.08^{\mathrm{a}}$ & $0.27 \pm 0.00^{\mathrm{a}}$ & $0.33 \pm 0.02^{\mathrm{a}}$ \\
\hline $16: 3 n-4$ & $0.17 \pm 0.03^{\mathrm{ab}}$ & $0.20 \pm 0.06^{\mathrm{a}}$ & $0.16 \pm 0.02^{\mathrm{ab}}$ & $0.14 \pm 0.03^{\mathrm{abc}}$ & $0.10 \pm 0.00^{\mathrm{c}}$ & $0.12 \pm 0.01^{\mathrm{bc}}$ \\
\hline $16: 3 n-3$ & $5.10 \pm 0.38^{\mathrm{a}}$ & $4.54 \pm 0.47^{\mathrm{ab}}$ & $4.45 \pm 0.62^{\mathrm{ab}}$ & $3.46 \pm 0.78^{\mathrm{b}}$ & $3.66 \pm 0.07^{\mathrm{b}}$ & $4.17 \pm 0.21^{\mathrm{ab}}$ \\
\hline $16: 4 n-3$ & $0.00 \pm 0.00^{\mathrm{a}}$ & $0.00 \pm 0.00^{\mathrm{a}}$ & $0.00 \pm 0.00^{\mathrm{a}}$ & $0.00 \pm 0.00^{\mathrm{a}}$ & $0.01 \pm 0.00^{\mathrm{a}}$ & $0.02 \pm 0.00^{\mathrm{a}}$ \\
\hline $18: 2 n-6$ & $0.85 \pm 0.20^{\mathrm{a}}$ & $0.40 \pm 0.01^{\mathrm{ab}}$ & $0.50 \pm 0.30^{\mathrm{ab}}$ & $0.31 \pm 0.07^{\mathrm{ab}}$ & $0.25 \pm 0.01^{\mathrm{b}}$ & $0.29 \pm 0.01^{\mathrm{ab}}$ \\
\hline $18: 3 n-6$ & $0.21 \pm 0.05^{\mathrm{a}}$ & $0.10 \pm 0.01^{\mathrm{b}}$ & $0.00 \pm 0.00^{\mathrm{b}}$ & $0.07 \pm 0.01^{\mathrm{b}}$ & $0.05 \pm 0.00^{\mathrm{b}}$ & $0.07 \pm 0.00^{\mathrm{b}}$ \\
\hline $18: 3 n-4$ & $0.12 \pm 0.01^{\mathrm{a}}$ & $0.00 \pm 0.00^{\mathrm{a}}$ & $0.00 \pm 0.00^{\mathrm{a}}$ & $0.03 \pm 0.00^{\mathrm{a}}$ & $0.00 \pm 0.00^{\mathrm{a}}$ & $0.00 \pm 0.00^{\mathrm{a}}$ \\
\hline $18: 3 n-3$ & $1.60 \pm 0.50^{\mathrm{a}}$ & $0.38 \pm 0.01^{\mathrm{b}}$ & $0.22 \pm 0.05^{\mathrm{b}}$ & $0.35 \pm 0.09^{\mathrm{b}}$ & $0.23 \pm 0.02^{\mathrm{b}}$ & $0.44 \pm 0.01^{\mathrm{b}}$ \\
\hline $18: 4 n-3$ & $2.14 \pm 0.73^{\mathrm{a}}$ & $0.77 \pm 0.01^{\mathrm{b}}$ & $0.34 \pm 0.07^{\mathrm{b}}$ & $0.54 \pm 0.20^{\mathrm{b}}$ & $0.40 \pm 0.02^{\mathrm{b}}$ & $0.65 \pm 0.06^{\mathrm{b}}$ \\
\hline $20: 2 n-6$ & $1.44 \pm 0.20^{\mathrm{a}}$ & $0.78 \pm 0.04^{\mathrm{b}}$ & $0.65 \pm 0.11^{\mathrm{bc}}$ & $0.52 \pm 0.14^{\mathrm{bc}}$ & $0.41 \pm 0.01^{\mathrm{c}}$ & $0.44 \pm 0.02^{\mathrm{c}}$ \\
\hline $20: 4 n-6$ & $1.86 \pm 0.43^{\mathrm{ab}}$ & $2.77 \pm 0.03^{\mathrm{a}}$ & $1.90 \pm 0.46^{\mathrm{ab}}$ & $1.77 \pm 0.56^{\mathrm{b}}$ & $1.53 \pm 0.10^{\mathrm{b}}$ & $2.05 \pm 0.05^{\mathrm{ab}}$ \\
\hline $20: 3 n-3$ & $0.18 \pm 0.04^{\mathrm{a}}$ & $0.04 \pm 0.00^{\mathrm{b}}$ & $0.00 \pm 0.00^{\mathrm{b}}$ & $0.03 \pm 0.02^{\mathrm{b}}$ & $0.02 \pm 0.00^{\mathrm{b}}$ & $0.00 \pm 0.00^{\mathrm{b}}$ \\
\hline $20: 4 n-3$ & $0.69 \pm 0.18^{\mathrm{a}}$ & $0.32 \pm 0.00^{\mathrm{ab}}$ & $0.17 \pm 0.02^{\mathrm{ab}}$ & $0.15 \pm 0.05^{\mathrm{ab}}$ & $0.13 \pm 0.00^{\mathrm{b}}$ & $0.19 \pm 0.01^{\mathrm{ab}}$ \\
\hline $20: 5 n-3$ & $8.38 \pm 2.48^{\mathrm{a}}$ & $5.35 \pm 0.16^{\mathrm{ab}}$ & $2.93 \pm 0.69^{\mathrm{bc}}$ & $2.55 \pm 0.86^{\mathrm{bc}}$ & $2.19 \pm 0.06^{\mathrm{c}}$ & $3.17 \pm 0.11^{\mathrm{bc}}$ \\
\hline $21: 5 n-3$ & $1.62 \pm 0.30^{\mathrm{a}}$ & $1.32 \pm 0.28^{\mathrm{ab}}$ & $1.03 \pm 0.12^{\mathrm{b}}$ & $0.92 \pm 0.27^{\mathrm{b}}$ & $0.94 \pm 0.02^{\mathrm{b}}$ & $1.37 \pm 0.02^{\mathrm{ab}}$ \\
\hline $22: 4 n-6$ & $1.08 \pm 0.13^{\mathrm{a}}$ & $1.57 \pm 0.06^{\mathrm{b}}$ & $1.03 \pm 0.23^{\mathrm{ac}}$ & $0.95 \pm 0.31^{\mathrm{ac}}$ & $0.93 \pm 0.01^{\mathrm{ac}}$ & $1.16 \pm 0.02^{\mathrm{abc}}$ \\
\hline $22: 5 n-6$ & $0.51 \pm 0.10^{\mathrm{a}}$ & $0.43 \pm 0.02^{\mathrm{a}}$ & $0.43 \pm 0.10^{\mathrm{a}}$ & $0.36 \pm 0.12^{\mathrm{a}}$ & $0.34 \pm 0.00^{\mathrm{a}}$ & $0.60 \pm 0.01^{\mathrm{a}}$ \\
\hline $22: 5 n-3$ & $2.20 \pm 0.42^{\mathrm{a}}$ & $1.94 \pm 0.06^{\mathrm{ab}}$ & $1.26 \pm 0.34^{\mathrm{bc}}$ & $1.08 \pm 0.38^{\mathrm{c}}$ & $1.14 \pm 0.02^{\mathrm{c}}$ & $1.76 \pm 0.02^{\mathrm{abc}}$ \\
\hline
\end{tabular}


Table 3 continued

\begin{tabular}{|c|c|c|c|c|c|c|}
\hline \multirow[t]{2}{*}{ Fatty acids } & \multirow{2}{*}{$\begin{array}{l}\text { Spring } \\
\text { April }\end{array}$} & \multicolumn{2}{|l|}{ Summer } & \multirow{2}{*}{$\begin{array}{l}\text { Autumn } \\
\text { October }\end{array}$} & \multicolumn{2}{|l|}{ Winter } \\
\hline & & June & August & & December & February \\
\hline $22: 6 n-3$ & $9.27 \pm 2.10^{\mathrm{a}}$ & $7.54 \pm 0.15^{\mathrm{ab}}$ & $5.48 \pm 1.35^{\mathrm{b}}$ & $4.67 \pm 1.65^{\mathrm{b}}$ & $4.49 \pm 0.06^{\mathrm{b}}$ & $6.03 \pm 0.05^{\mathrm{ab}}$ \\
\hline$\sum$ PUFA & $37.61 \pm 8.24^{\mathrm{a}}$ & $28.41 \pm 1.43^{\mathrm{a}}$ & $20.80 \pm 3.87^{\mathrm{b}}$ & $18.11 \pm 5.59^{\mathrm{b}}$ & $17.06 \pm 0.36^{\mathrm{b}}$ & $22.82 \pm 0.32^{\mathrm{b}}$ \\
\hline$\sum n-3$ & $31.18 \pm 7.11^{\mathrm{a}}$ & $21.91 \pm 1.32^{\mathrm{ab}}$ & $15.88 \pm 3.23^{\mathrm{ab}}$ & $13.76 \pm 4.28^{\mathrm{ab}}$ & $13.21 \pm 0.23^{\mathrm{b}}$ & $17.80 \pm 0.34^{\mathrm{ab}}$ \\
\hline$\sum n-6$ & $5.94 \pm 1.10^{\mathrm{a}}$ & $6.02 \pm 0.12^{\mathrm{a}}$ & $4.51 \pm 0.61^{\mathrm{ab}}$ & $3.95 \pm 1.23^{\mathrm{b}}$ & $3.49 \pm 0.13^{b}$ & $4.56 \pm 0.07^{\mathrm{ab}}$ \\
\hline$n-3 / n-6$ & $5.21 \pm 0.25^{\mathrm{a}}$ & $3.64 \pm 0.20^{\mathrm{b}}$ & $3.50 \pm 0.28^{\mathrm{b}}$ & $3.49 \pm 0.03^{\mathrm{b}}$ & $3.79 \pm 0.09^{b}$ & $3.90 \pm 0.13^{\mathrm{b}}$ \\
\hline DHA/EPA & $1.13 \pm 0.10^{\mathrm{a}}$ & $1.41 \pm 0.04^{\mathrm{b}}$ & $1.87 \pm 0.04^{\mathrm{c}}$ & $1.82 \pm 0.04^{\mathrm{c}}$ & $2.05 \pm 0.03^{\mathrm{d}}$ & $1.90 \pm 0.08^{\mathrm{cd}}$ \\
\hline$\sum F A$ & $87.94 \pm 18.15^{\mathrm{a}}$ & $62.49 \pm 2.2^{\mathrm{b}}$ & $39.76 \pm 6.46^{\mathrm{bc}}$ & $34.53 \pm 9.97^{\mathrm{c}}$ & $34.02 \pm 0.78^{\mathrm{c}}$ & $42.09 \pm 1.65^{\mathrm{bc}}$ \\
\hline
\end{tabular}

Different superscript letters within rows represent significant differences $(P<0.05)$ and should be interpreted in the following exemplificative manner: $\mathbf{a}$ not $\neq \mathbf{a}, \mathbf{a} \neq \mathbf{b}, \mathbf{a} \neq \mathbf{c} ; \mathbf{a b} \neq \mathbf{c d}, \mathbf{a}$ not $\neq \mathbf{a b}, \mathbf{a b}$ not $\neq \mathbf{b c}$

Values are means of triplicate samples $( \pm \mathrm{SD})$

Fig. 3 Main saturated fatty acid, main monounsaturated fatty acid and respective fractions (SFA, MUFA) contents between April 2010 and February 2011. A Palmitic acid, $\mathbf{B}$ stearic acid, $\mathbf{C}$ saturated fatty acid fraction, $\mathbf{D}$ oleic acid, E vaccenic acid,

F monounsaturated fatty acid fraction. Values are means of triplicate samples $( \pm \mathrm{SD})$. Different superscript letters represent significant differences between months $(P<0.05)$ and should be interpreted as exemplified in Fig. 1 caption. Temporal evolution in gonad development stages as determined by Gaspar and Monteiro (1998) is presented
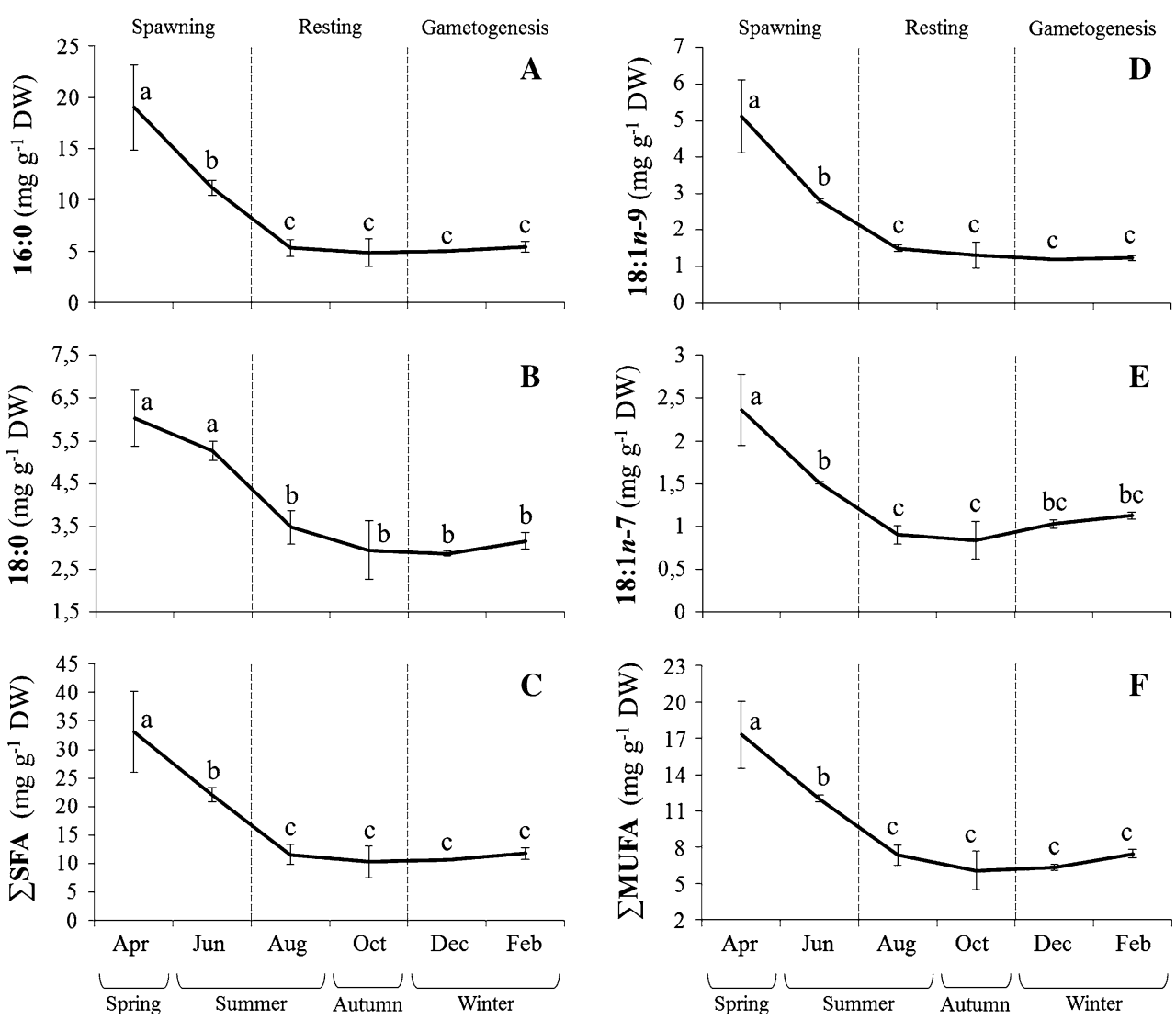

April and December (Fig. 2D, Tukey's test, $F=7.10$, $\mathrm{df}=5, P<0.01)$. The complete AA profile of E. siliqua is shown in Table 2.

Seasonal variations in fatty acid composition

The general trend among FAs exhibited one peak in April and a minimum either in October or December. A few different trends were, however, observed (Table 3).
The main saturated fatty acids (SFAs) were palmitic (16:0) and stearic (18:0; Fig. 3B) acids. Content of 16:0 was significantly greater in April when compared to all other months (Fig. 3A, Tukey's test, $F=30.56$, df $=5$, $P<0.01$ ). SFA fraction ( $\sum$ SFA) levels were significantly higher in April when compared to all other months (Fig. 3C, Tukey's test, $F=24.76$, df $=5, P<0.01$ ).

The main monounsaturated fatty acids (MUFAs) were oleic $(18: 1 n-9), \quad$ vaccenic $(18: 1 n-7 ; \quad$ Fig. $3 \mathrm{E})$ and 
Fig. 4 Main polyunsaturated fatty acid, respective fraction (PUFA) and total fatty acid contents between April 2010 and February 2011.

A Hexadecatrienoic acid,

B $\alpha$-linolenic acid,

C eicosapentaenoic acid,

D adrenic acid,

E polyunsaturated fatty acid fraction, $\mathbf{F}$ total fatty acid content. Values are means of triplicate samples $( \pm \mathrm{SD})$.

Different superscript letters represent significant differences between months $(P<0.05)$ and should be interpreted as exemplified in Fig. 1 caption. Temporal evolution in gonad development stages as determined by Gaspar and Monteiro (1998) is presented
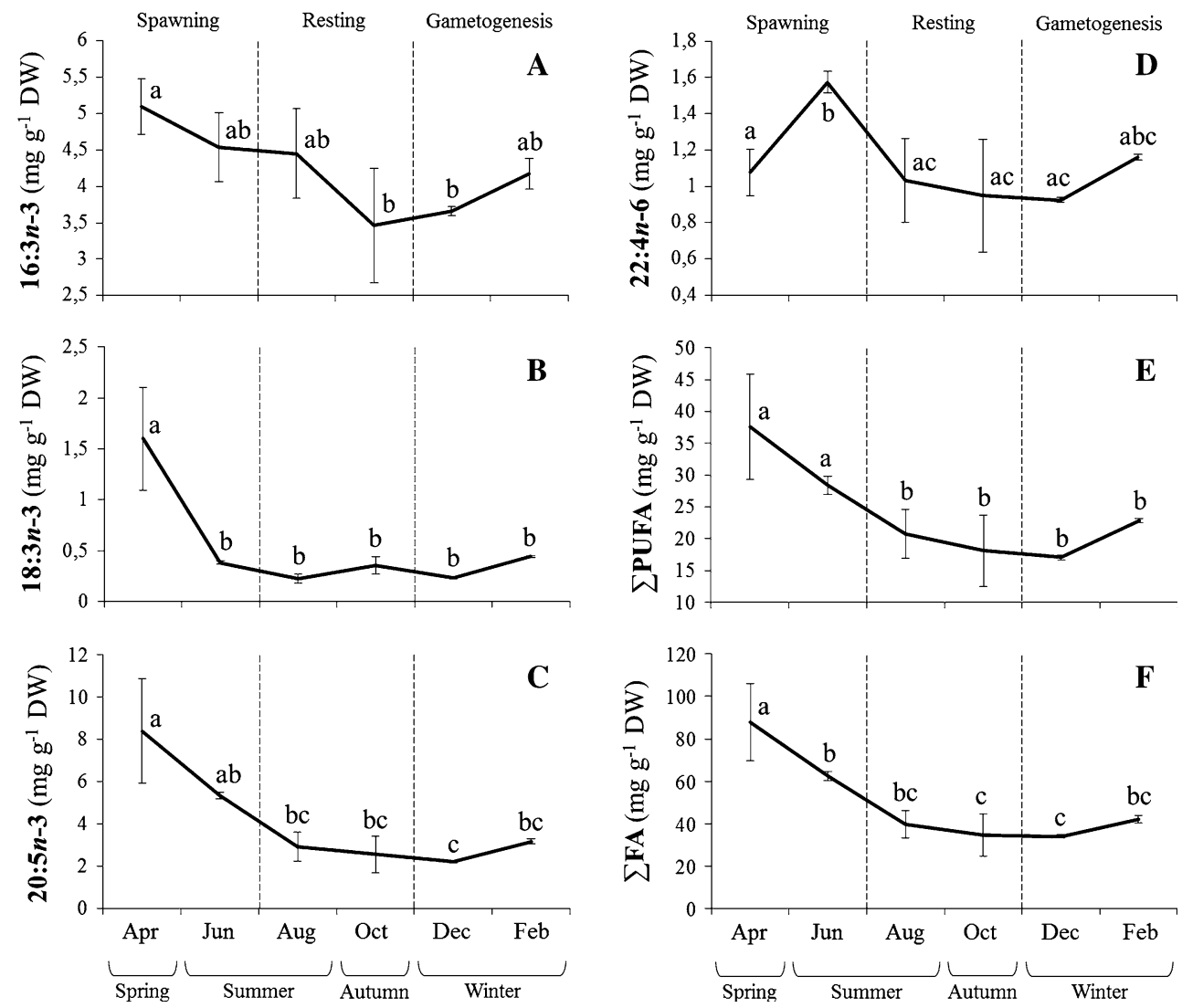

palmitoleic (16:1n-7) acids. 18:1n-9 content was significantly greater in April when compared to all other months (Fig. 3D, Tukey's test, $F=38.65$, df $=5, P<0.01$ ). The content of 16:1n-7 surpassed that of 18:1n-7 in April and June. Levels of $16: 1 n-7$ were also significantly higher in April when compared to all other months (Table 3, Tukey's test, $F=51.16$, df $=5, P<0.01)$. Concomitantly, MUFA fraction ( $\sum$ MUFA) content was significantly greater in April when compared to all other months (Fig. 3F, Tukey's test, $F=31.22$, df $=5, P<0.01$ ).

Among polyunsaturated fatty acids (PUFAs), the seasonal trends varied considerably. The $n-3$ family dominated this fraction. The main PUFAs were docosahexaenoic (DHA, 22:6n-3), hexadecatrienoic (16:3n-3; Fig. 4A) and eicosapentaenoic (EPA, 20:5n-3) acids. 16:3n-3 was the second main PUFA except in April and June, when it was surpassed by 20:5n-3. 20:5n-3 exhibited significantly higher values in April when compared to those obtained between August and December (Fig. 4C, Tukey's test, $F=13.73$, df $=5, P<0.01)$. Regarding $\alpha$-linolenic acid (18:3n-3), a significantly higher value was observed in April when compared to all other months (Fig. 4B, Tukey's test, $F=18.35$, df $=5, P<0.01)$. On the other hand, adrenic acid (22:4n-6) content was significantly greater in June when compared to all other months (Fig. 4D, Tukey's test, $F=5.96$, df $=5, P<0.01)$. Differently from $\sum \mathrm{SFA}$ and $\sum$ MUFA, PUFA fraction ( $\sum$ PUFA) levels were significantly higher in April and June when compared to all other months (Fig. 4E, Kruskal-Wallis, $H=9.26$, df $=5$, $P<0.01)$. Finally, total FA $\left(\sum \mathrm{FA}\right)$ content was significantly greater in April when compared to all other months (Fig. 4F, Tukey's test, $F=16.98$, df $=5, P<0.01$ ). The complete FA profile of E. siliqua is shown in Table 3.

Environmental parameters and biochemical composition

Mean turbidity (here as a proxy of food availability) peaked in spring (Kruskal-Wallis, $H=8.90$, df $=3$, $P<0.05$; Fig. 5A). Mean salinity did not vary significantly throughout seasons (Kruskal-Wallis, $H=3.36$, df $=3, P>0.05$; Fig. 5B). Mean water temperature in Caldeira de Tróia varied from $15^{\circ} \mathrm{C}$ in winter to $20^{\circ} \mathrm{C}$ in summer (Kruskal-Wallis, $H=9.34$, df $=3, P<0.05$; Fig. 5C). There was no significant relationship between environmental parameters (Table 1). Moreover, none of the three environmental parameters measured (i.e., turbidity, salinity and water temperature), nor a combination between parameters, had a significant effect on amino acid or fatty acid composition of E. siliqua (see the low $R^{2}$ values irrespective of significance levels in Tables 2 and 3). 

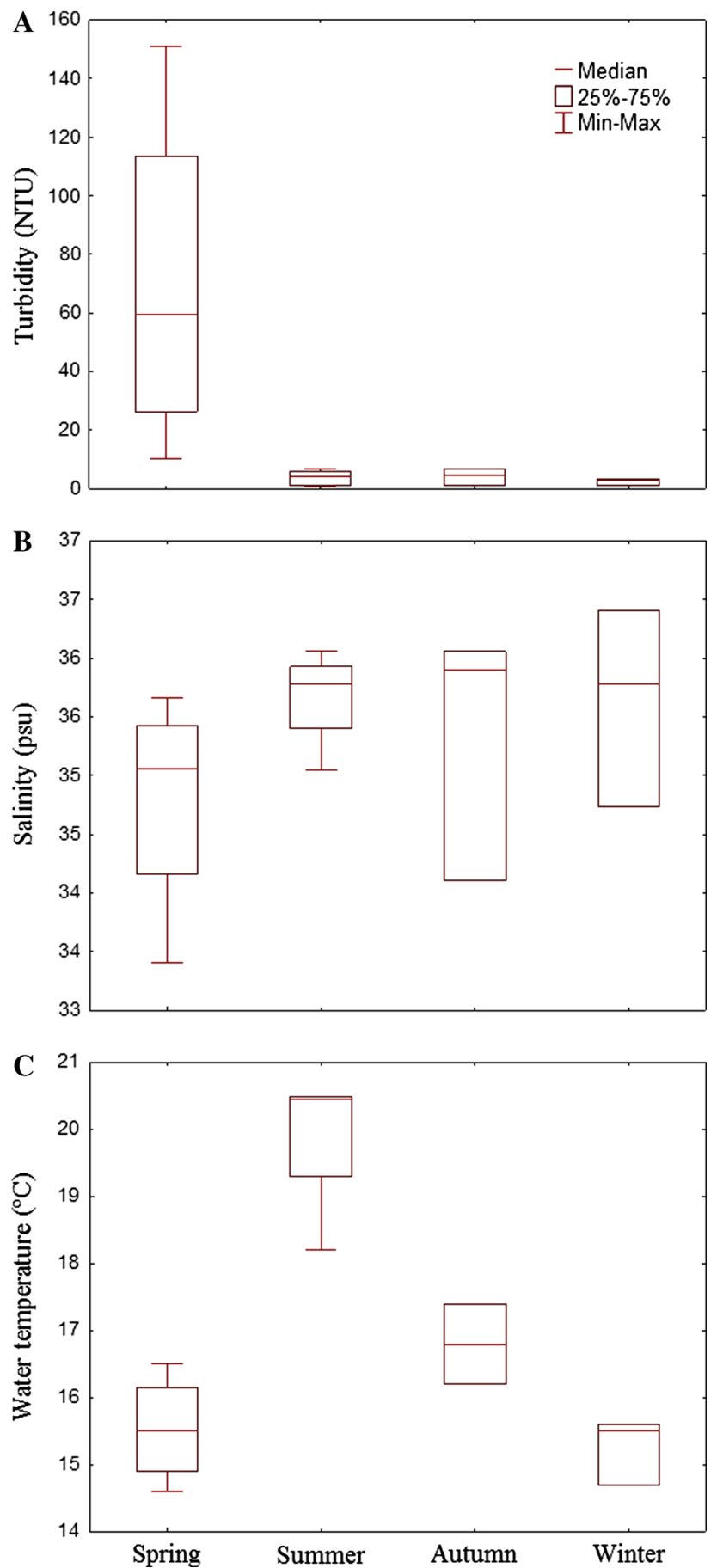

Fig. 5 Seasonal changes of biotic and abiotic factors in Caldeira de Tróia obtained from September 2007 to September 2010. A Turbidity (NTU, as a proxy of food availability), B salinity (psu), C temperature $\left({ }^{\circ} \mathrm{C}\right)$. Spring: 1 March-31 May; Summer: 1 June-31 August; Autumn: 1 September-30 November; Winter: 1 December-28 February

\section{Discussion}

The biochemical composition of an organism is determined by endogenous processes (e.g., gametogenesis, maturation, spawning) and exogenous factors (e.g., food availability, salinity, temperature) (Rosa et al. 2002; Rosa and Nunes 2003; Ojea et al. 2004; Baptista et al. 2012). A temporal analysis of the biochemical compounds permits intercrossing with chronological data of other variables allowing the researcher to gather knowledge on the ecology and physiology of an organism and also to understand how the surrounding environment may affect it.

Studies dealing with both biochemical composition and gametogenesis of bivalves do not usually examine the extent of the differential loss of biochemical content by males and females (through release of gametes) in gonochoric species, or analogously, by the male and female reproductive tissues in hermaphroditic species (e.g., Barber and Blake 1981; Ojea et al. 2004; Tarnowska et al. 2009; Yang et al. 2011). Hayward and Gillooly (2011) studied the cost of gamete production for a broad variety of invertebrate and vertebrate organisms and found that although males and females (of the same species) invest similarly in gonad biomass, gamete biomass production rate is approximately two to four orders of magnitude higher in females. While studying E. siliqua, a sex ratio of 1:1 was observed in populations from the south of Portugal (Gaspar and Monteiro 1998) and northwest Spain (Darriba et al. 2005b; da Costa et al. 2010); thus, it is reasonable to assume that a similar ratio should occur among the population of Caldeira de Tróia. Still, even if such ratio is not verified, the immensely greater accumulation (during gametogenesis) and subsequent loss (through spawning) of $\mathrm{AA}$ and FA reserves (in eggs) by females should be regarded as essentially responsible for the AA and FA fluctuations observed in the present study.

Amino acid profile and seasonality

The EAAs found in greater quantity were arginine, leucine and lysine while the main NEAAs, and overall main AAs, were glutamic acid, glycine and aspartic acid. Upon comparison of these results with those obtained in other studies, it appears that bivalve AA composition varies considerably between species. Sidwell et al. (1979) found that the main AAs in Crassostrea virginica were glutamic acid, aspartic acid and lysine. On the other hand, Özden and Erkan (2011) studied Flexopecten glaber and found the main AAs to be proline, glutamic acid, phenylalanine and aspartic acid. Still, considering those two studies and the present one, it would appear that glutamic acid and aspartic acid are always found among the main AAs. In the study of Fatima (1996), however, the main AAs of Perna viridis were found to be arginine, leucine and lysine while glutamic acid and aspartic acid were the AAs found in smaller concentration. In terms of $\sum A A$ content, values between 46.94 and $54.67 \%$ dry weight (DW) were obtained for $E$. 
Table 4 Models assessing the importance of environmental parameters in predicting amino acid composition of E. siliqua in Caldeira de Tróia

\begin{tabular}{|c|c|c|c|c|c|}
\hline Amino acids & Model & $\begin{array}{l}\text { Turbidity } \\
\text { ( } t \text { ratio) }\end{array}$ & $\begin{array}{l}\text { Salinity } \\
\text { ( } t \text { ratio })\end{array}$ & $\begin{array}{l}\text { SST } \\
\text { ( } t \text { ratio) }\end{array}$ & $\begin{array}{l}\text { Total } \\
\mathrm{R}^{2}\end{array}$ \\
\hline \multicolumn{6}{|c|}{ Essential (EAA) } \\
\hline \multirow[t]{4}{*}{ THR } & 1 & -1.92 & & & 0.19 \\
\hline & 2 & & $2.48 *$ & & 0.28 \\
\hline & 3 & & & 1.55 & 0.13 \\
\hline & 4 & & $2.41 *$ & 1.51 & 0.37 \\
\hline \multirow[t]{4}{*}{ VAL } & 1 & -1.29 & & & 0.09 \\
\hline & 2 & & $2.44 *$ & & 0.27 \\
\hline & 3 & & & 1.88 & 0.18 \\
\hline & 4 & & $2.42 *$ & 1.89 & 0.41 \\
\hline \multirow[t]{4}{*}{ MET } & 1 & -1.25 & & & 0.09 \\
\hline & 2 & & 0.90 & & 0.05 \\
\hline & 3 & & & 1.20 & 0.08 \\
\hline & 4 & -0.97 & & 0.91 & 0.14 \\
\hline \multirow[t]{4}{*}{ ILE } & 1 & $-2.14 *$ & & & 0.22 \\
\hline & 2 & & $2.79 *$ & & 0.33 \\
\hline & 3 & & & 1.95 & 0.19 \\
\hline & 4 & & $2.83 *$ & 2.04 & 0.47 \\
\hline \multirow[t]{4}{*}{ LEU } & 1 & $-2.24 *$ & & & 0.24 \\
\hline & 2 & & $2.62 *$ & & 0.30 \\
\hline & 3 & & & $2.25 *$ & 0.24 \\
\hline & 4 & & $2.71 *$ & $2.36^{*}$ & 0.49 \\
\hline \multirow[t]{4}{*}{ PHE } & 1 & -1.49 & & & 0.12 \\
\hline & 2 & & $2.39 *$ & & 0.26 \\
\hline & 3 & & & 1.82 & 0.17 \\
\hline & 4 & & $2.35^{*}$ & 1.81 & 0.40 \\
\hline \multirow[t]{4}{*}{ HIS } & 1 & -0.26 & & & 0.00 \\
\hline & 2 & & 1.26 & & 0.09 \\
\hline & 3 & & & 1.29 & 0.09 \\
\hline & 4 & & 1.15 & 1.18 & 0.17 \\
\hline \multirow[t]{4}{*}{ LYS } & 1 & -0.70 & & & 0.03 \\
\hline & 2 & & 0.99 & & 0.06 \\
\hline & 3 & & & 1.62 & 0.14 \\
\hline & 4 & & 0.86 & 1.51 & 0.18 \\
\hline \multirow[t]{4}{*}{ ARG } & 1 & -0.76 & & & 0.03 \\
\hline & 2 & & 1.26 & & 0.09 \\
\hline & 3 & & & $2.90 *$ & 0.34 \\
\hline & 4 & & 1.21 & $2.80 *$ & 0.40 \\
\hline \multirow[t]{4}{*}{$\sum \mathrm{EAA}$} & 1 & -1.46 & & & 0.12 \\
\hline & 2 & & 2.02 & & 0.20 \\
\hline & 3 & & & $2.46^{*}$ & 0.27 \\
\hline & 4 & & 2.05 & $2.47 *$ & 0.43 \\
\hline
\end{tabular}

siliqua. These values are in agreement with total protein content found for other marine bivalves, namely, Siliqua patula, a species belonging to the same family as E. siliqua
Table 4 continued

\begin{tabular}{|c|c|c|c|c|c|}
\hline Amino acids & Model & $\begin{array}{l}\text { Turbidity } \\
(t \text { ratio })\end{array}$ & $\begin{array}{l}\text { Salinity } \\
(t \text { ratio })\end{array}$ & $\begin{array}{l}\text { SST } \\
(t \text { ratio })\end{array}$ & $\begin{array}{l}\text { Total } \\
\mathrm{R}^{2}\end{array}$ \\
\hline \multicolumn{6}{|l|}{$\begin{array}{l}\text { Non-essential } \\
\text { (NEAA) }\end{array}$} \\
\hline \multirow[t]{4}{*}{ ASP } & 1 & $-2.37^{*}$ & & & 0.26 \\
\hline & 2 & & $2.77 *$ & & 0.32 \\
\hline & 3 & & & $2.82 *$ & 0.33 \\
\hline & 4 & & $3.12 * *$ & $3.16^{* *}$ & 0.59 \\
\hline \multirow[t]{4}{*}{ TYR } & 1 & -1.98 & & & 0.20 \\
\hline & 2 & & $2.34 *$ & & 0.25 \\
\hline & 3 & & & $2.16^{*}$ & 0.23 \\
\hline & 4 & & $2.36^{*}$ & $2.19 *$ & 0.43 \\
\hline \multirow[t]{4}{*}{ SER } & 1 & -0.33 & & & 0.01 \\
\hline & 2 & & 1.81 & & 0.17 \\
\hline & 3 & & & 2.06 & 0.21 \\
\hline & 4 & & 1.75 & 1.99 & 0.34 \\
\hline \multirow[t]{4}{*}{ GLU } & 1 & $-2.42 *$ & & & 0.27 \\
\hline & 2 & & $2.78^{*}$ & & 0.33 \\
\hline & 3 & & & $2.77 *$ & 0.32 \\
\hline & 4 & & $3.11 * *$ & $3.10 * *$ & 0.59 \\
\hline \multirow[t]{4}{*}{ GLY } & 1 & -0.29 & & & 0.01 \\
\hline & 2 & & 0.60 & & 0.02 \\
\hline & 3 & & & $4.05^{* *}$ & 0.51 \\
\hline & 4 & 0.64 & & $4.01 * *$ & 0.52 \\
\hline \multirow[t]{4}{*}{ ALA } & 1 & 1.08 & & & 0.07 \\
\hline & 2 & & 0.51 & & 0.02 \\
\hline & 3 & & & $2.21 *$ & 0.23 \\
\hline & 4 & 1.98 & & $2.83 *$ & 0.39 \\
\hline \multirow[t]{4}{*}{ PRO } & 1 & -1.06 & & & 0.07 \\
\hline & 2 & & $2.48 *$ & & 0.28 \\
\hline & 3 & & & 1.54 & 0.13 \\
\hline & 4 & & $2.40 *$ & 1.49 & 0.37 \\
\hline \multirow[t]{4}{*}{$\sum \mathrm{NEAA}$} & 1 & -1.30 & & & 0.10 \\
\hline & 2 & & $2.14 *$ & & 0.22 \\
\hline & 3 & & & $4.15^{* *}$ & 0.52 \\
\hline & 4 & & $2.72 *$ & $4.60 * *$ & 0.68 \\
\hline \multirow[t]{4}{*}{$\sum \mathrm{AA}$} & 1 & -1.42 & & & 0.11 \\
\hline & 2 & & $2.17 *$ & & 0.23 \\
\hline & 3 & & & $3.47 * *$ & 0.43 \\
\hline & 4 & & $2.51 *$ & $3.72 * *$ & 0.60 \\
\hline
\end{tabular}

For each variable, the $t$ ratios are included. Models $1-4$ evaluated the following: 1: turbidity; 2: salinity; 3: water temperature; 4: more explanatory pair of environmental parameters. $* P<0.05$, ** $P<0.01$

(i.e., Pharidae) (46.0-48.0 \% DW; Lewin et al. 1979), Venerupis decussata (44.7-50.8 DW; Ojea et al. 2004), $P$. viridis (57.8-66.5\% DW; Fatima 1996), C. virginica (32.5-70.1 \% DW; Sidwell et al. 1979) and Cerastoderma glaucum (37.5-77.4\% DW; Tarnowska et al. 2009). 
Table 5 Models assessing the importance of environmental parameters in predicting fatty acid composition of E. siliqua in Caldeira de Tróia

\begin{tabular}{|c|c|c|c|c|c|}
\hline Fatty acids & Model & Turbidity $(t$ ratio) & Salinity $(t$ ratio $)$ & SST ( $t$ ratio) & $\overline{\text { Total } R^{2}}$ \\
\hline \multicolumn{6}{|l|}{ Saturated (SFA) } \\
\hline \multirow[t]{4}{*}{$14: 0$} & 1 & $4.62 * *$ & & & 0.57 \\
\hline & 2 & & -1.02 & & 0.06 \\
\hline & 3 & & & -0.21 & 0.00 \\
\hline & 4 & $4.66^{* *}$ & & 0.88 & 0.59 \\
\hline \multirow[t]{4}{*}{$15: 0$} & 1 & $3.86^{* *}$ & & & 0.48 \\
\hline & 2 & & -0.82 & & 0.04 \\
\hline & 3 & & & -0.63 & 0.02 \\
\hline & 4 & $3.60^{* *}$ & 0.31 & & 0.48 \\
\hline \multirow[t]{4}{*}{$16: 0$} & 1 & $5.04 * *$ & & & 0.61 \\
\hline & 2 & & -1.28 & & 0.09 \\
\hline & 3 & & & -0.32 & 0.01 \\
\hline & 4 & $5.03 * *$ & & 0.80 & 0.63 \\
\hline \multirow[t]{4}{*}{$17: 0$} & 1 & $3.20 * *$ & & & 0.39 \\
\hline & 2 & & -0.88 & & 0.05 \\
\hline & 3 & & & -0.07 & 0.00 \\
\hline & 4 & $3.24 * *$ & & 0.74 & 0.41 \\
\hline \multirow[t]{4}{*}{ 18:0 } & 1 & $3.42 * *$ & & & 0.42 \\
\hline & 2 & & -1.07 & & 0.07 \\
\hline & 3 & & & 0.62 & 0.02 \\
\hline & 4 & $4.03^{* *}$ & & 1.87 & 0.53 \\
\hline \multirow[t]{4}{*}{ 19:0 } & 1 & $2.77^{*}$ & & & 0.32 \\
\hline & 2 & & -0.85 & & 0.04 \\
\hline & 3 & & & 0.65 & 0.03 \\
\hline & 4 & $3.22 * *$ & & 1.62 & 0.42 \\
\hline \multirow[t]{4}{*}{$20: 0$} & 1 & 0.41 & & & 0.01 \\
\hline & 2 & & 0.85 & & 0.04 \\
\hline & 3 & & & 0.54 & 0.02 \\
\hline & 4 & 0.78 & 1.07 & & 0.08 \\
\hline \multirow[t]{4}{*}{$22: 0$} & 1 & 1.33 & & & 0.10 \\
\hline & 2 & & -0.55 & & 0.02 \\
\hline & 3 & & & -0.45 & 0.01 \\
\hline & 4 & 1.21 & & -0.13 & 0.10 \\
\hline \multirow[t]{4}{*}{ Iso $15: 0$} & 1 & $2.57^{*}$ & & & 0.29 \\
\hline & 2 & & -0.14 & & 0.00 \\
\hline & 3 & & & -0.23 & 0.00 \\
\hline & 4 & $2.67^{*}$ & 0.82 & & 0.32 \\
\hline \multirow[t]{4}{*}{ Anteiso 16:0 } & 1 & 1.67 & & & 0.15 \\
\hline & 2 & & -0.65 & & 0.03 \\
\hline & 3 & & & -1.45 & 0.12 \\
\hline & 4 & 1.35 & & -1.09 & 0.21 \\
\hline \multirow[t]{4}{*}{ Iso $17: 0$} & 1 & $4.29 * *$ & & & 0.54 \\
\hline & 2 & & -1.19 & & 0.08 \\
\hline & 3 & & & -0.73 & 0.03 \\
\hline & 4 & $3.83^{* *}$ & -0.10 & & 0.54 \\
\hline \multirow[t]{4}{*}{$\sum \mathrm{SFA}$} & 1 & $4.59^{* *}$ & & & 0.57 \\
\hline & 2 & & -1.17 & & 0.08 \\
\hline & 3 & & & -0.14 & 0.00 \\
\hline & 4 & $4.68 * *$ & & 0.99 & 0.59 \\
\hline
\end{tabular}


Table 5 continued

\begin{tabular}{|c|c|c|c|c|c|}
\hline Fatty acids & Model & Turbidity ( $t$ ratio) & Salinity ( $t$ ratio) & SST ( $t$ ratio) & Total $R^{2}$ \\
\hline \multicolumn{6}{|c|}{ Monounsaturated (MUFA) } \\
\hline \multirow[t]{4}{*}{$16: 1 n-9$} & 1 & 0.64 & & & 0.02 \\
\hline & 2 & & -0.45 & & 0.01 \\
\hline & 3 & & & -1.20 & 0.08 \\
\hline & 4 & 0.35 & & -1.03 & 0.09 \\
\hline \multirow[t]{4}{*}{$16: 1 n-7$} & 1 & $3.78^{* *}$ & & & 0.47 \\
\hline & 2 & & -1.05 & & 0.06 \\
\hline & 3 & & & 0.26 & 0.00 \\
\hline & 4 & $4.12 * *$ & & 1.40 & 0.53 \\
\hline \multirow[t]{4}{*}{$16: 1 n-5$} & 1 & $3.63 * *$ & & & 0.45 \\
\hline & 2 & & -1.22 & & 0.09 \\
\hline & 3 & & & 0.51 & 0.02 \\
\hline & 4 & $4.20 * *$ & & 1.78 & 0.55 \\
\hline \multirow[t]{4}{*}{$17: 1$} & 1 & 1.94 & & & 0.19 \\
\hline & 2 & & -0.34 & & 0.01 \\
\hline & 3 & & & -0.13 & 0.00 \\
\hline & 4 & 1.92 & & 0.36 & 0.20 \\
\hline \multirow[t]{4}{*}{$18: 1 n-9$} & 1 & $5.27 * *$ & & & 0.63 \\
\hline & 2 & & -1.43 & & 0.11 \\
\hline & 3 & & & -0.16 & 0.00 \\
\hline & 4 & $5.42 * *$ & & 1.12 & 0.66 \\
\hline \multirow[t]{4}{*}{$18: 1 n-7$} & 1 & $4.99 * *$ & & & 0.61 \\
\hline & 2 & & -1.34 & & 0.10 \\
\hline & 3 & & & -0.71 & 0.03 \\
\hline & 4 & $4.43^{* *}$ & -0.22 & & 0.61 \\
\hline \multirow[t]{4}{*}{$18: 1 n-5$} & 1 & -0.76 & & & 0.03 \\
\hline & 2 & & 0.92 & & 0.05 \\
\hline & 3 & & & 1.08 & 0.07 \\
\hline & 4 & & 0.82 & 0.98 & 0.11 \\
\hline \multirow[t]{4}{*}{$20: 1 n-11$} & 1 & 1.43 & & & 0.11 \\
\hline & 2 & & -0.54 & & 0.02 \\
\hline & 3 & & & $2.84 *$ & 0.33 \\
\hline & 4 & $3.02 * *$ & & $4.14 * *$ & 0.59 \\
\hline \multirow[t]{4}{*}{$20: 1 n-9$} & 1 & $5.50 * *$ & & & 0.65 \\
\hline & 2 & & -1.72 & & 0.16 \\
\hline & 3 & & & -0.19 & 0.00 \\
\hline & 4 & $5.65^{* *}$ & & 1.13 & 0.68 \\
\hline \multirow[t]{4}{*}{$20: 1 n-7$} & 1 & $2.16^{*}$ & & & 0.23 \\
\hline & 2 & & -0.56 & & 0.02 \\
\hline & 3 & & & -0.56 & 0.02 \\
\hline & 4 & 2.01 & 0.16 & & 0.23 \\
\hline \multirow[t]{4}{*}{$22: 1 n-9$} & 1 & -0.68 & & & 0.03 \\
\hline & 2 & & 1.61 & & 0.14 \\
\hline & 3 & & & -1.31 & 0.10 \\
\hline & 4 & & 1.83 & -1.58 & 0.26 \\
\hline \multirow[t]{4}{*}{$\sum$ MUFA } & 1 & $4.55^{* *}$ & & & 0.56 \\
\hline & 2 & & -1.28 & & 0.09 \\
\hline & 3 & & & -0.04 & 0.00 \\
\hline & 4 & $4.74 * *$ & & 1.15 & 0.60 \\
\hline
\end{tabular}


Table 5 continued

\begin{tabular}{|c|c|c|c|c|c|}
\hline Fatty acids & Model & Turbidity ( $t$ ratio) & Salinity ( $t$ ratio) & SST ( $t$ ratio) & Total $R^{2}$ \\
\hline \multicolumn{6}{|c|}{ Polyunsaturated (PUFA) } \\
\hline \multirow[t]{4}{*}{$16: 2 n-4$} & 1 & -1.04 & & & 0.06 \\
\hline & 2 & & -0.10 & & 0.00 \\
\hline & 3 & & & -0.74 & 0.03 \\
\hline & 4 & -1.27 & & -1.06 & 0.13 \\
\hline \multirow[t]{4}{*}{$16: 3 n-4$} & 1 & 0.74 & & & 0.03 \\
\hline & 2 & & -0.05 & & 0.00 \\
\hline & 3 & & & $2.81^{*}$ & 0.33 \\
\hline & 4 & 1.77 & & $3.34 * *$ & 0.45 \\
\hline \multirow[t]{4}{*}{$16: 3 n-3$} & 1 & $2.57 *$ & & & 0.29 \\
\hline & 2 & & -1.16 & & 0.08 \\
\hline & 3 & & & 1.02 & 0.06 \\
\hline & 4 & $3.27 * *$ & & 2.08 & 0.45 \\
\hline \multirow[t]{4}{*}{$16: 4 n-3$} & 1 & -0.84 & & & 0.04 \\
\hline & 2 & & 1.00 & & 0.06 \\
\hline & 3 & & & -1.89 & 0.18 \\
\hline & 4 & -1.50 & & $-2.28 *$ & 0.29 \\
\hline \multirow[t]{4}{*}{$18: 2 n-6$} & 1 & $3.78^{* *}$ & & & 0.47 \\
\hline & 2 & & -1.43 & & 0.11 \\
\hline & 3 & & & 0.41 & 0.01 \\
\hline & 4 & $4.28 * *$ & & 1.66 & 0.55 \\
\hline \multirow[t]{4}{*}{$18: 3 n-6$} & 1 & $4.61 * *$ & & & 0.57 \\
\hline & 2 & & -1.48 & & 0.12 \\
\hline & 3 & & & -0.93 & 0.05 \\
\hline & 4 & $4.02 * *$ & -0.45 & & 0.58 \\
\hline \multirow[t]{4}{*}{$18: 3 n-4$} & 1 & $3.96^{* *}$ & & & 0.49 \\
\hline & 2 & & -1.02 & & 0.06 \\
\hline & 3 & & & -1.19 & 0.08 \\
\hline & 4 & $3.59^{* *}$ & & -0.60 & 0.51 \\
\hline \multirow[t]{4}{*}{$18: 3 n-3$} & 1 & $5.97 * *$ & & & 0.69 \\
\hline & 2 & & -1.54 & & 0.13 \\
\hline & 3 & & & -1.34 & 0.10 \\
\hline & 4 & $5.50^{* *}$ & & -0.76 & 0.70 \\
\hline \multirow[t]{4}{*}{$18: 4 n-3$} & 1 & $5.49^{* *}$ & & & 0.65 \\
\hline & 2 & & -1.37 & & 0.11 \\
\hline & 3 & & & -1.28 & 0.09 \\
\hline & 4 & $5.04 * *$ & & -0.68 & 0.66 \\
\hline \multirow[t]{4}{*}{$20: 2 n-6$} & 1 & $5.25^{* *}$ & & & 0.63 \\
\hline & 2 & & -1.71 & & 0.16 \\
\hline & 3 & & & 0.12 & 0.00 \\
\hline & 4 & $5.78^{* *}$ & & 1.67 & 0.69 \\
\hline \multirow[t]{4}{*}{$20: 4 n-6$} & 1 & -0.24 & & & 0.00 \\
\hline & 2 & & 0.82 & & 0.04 \\
\hline & 3 & & & 1.65 & 0.15 \\
\hline & 4 & & 0.69 & 1.54 & 0.17 \\
\hline \multirow[t]{4}{*}{$20: 3 n-3$} & 1 & $7.01 * *$ & & & 0.75 \\
\hline & 2 & & $-2.11^{\mathrm{a}}$ & & 0.22 \\
\hline & 3 & & & -1.06 & 0.07 \\
\hline & 4 & $6.19^{* *}$ & -1.33 & & 0.78 \\
\hline \multirow[t]{4}{*}{$20: 4 n-3$} & 1 & $5.38^{* *}$ & & & 0.64 \\
\hline & 2 & & -1.38 & & 0.11 \\
\hline & 3 & & & -0.52 & 0.02 \\
\hline & 4 & $5.21 * *$ & & 0.51 & 0.65 \\
\hline
\end{tabular}


Table 5 continued

\begin{tabular}{|c|c|c|c|c|c|}
\hline Fatty acids & Model & Turbidity ( $t$ ratio) & Salinity ( $t$ ratio) & SST ( $t$ ratio) & Total $R^{2}$ \\
\hline \multirow[t]{4}{*}{$20: 5 n-3$} & 1 & $4.35^{* *}$ & & & 0.54 \\
\hline & 2 & & -0.99 & & 0.06 \\
\hline & 3 & & & -0.15 & 0.00 \\
\hline & 4 & $4.42 * *$ & & 0.92 & 0.57 \\
\hline \multirow[t]{4}{*}{$21: 5 n-3$} & 1 & $2.49^{*}$ & & & 0.28 \\
\hline & 2 & & -0.81 & & 0.04 \\
\hline & 3 & & & -0.74 & 0.03 \\
\hline & 4 & $2.28 *$ & & -0.22 & 0.28 \\
\hline \multirow[t]{4}{*}{$22: 4 n-6$} & 1 & -0.08 & & & 0.00 \\
\hline & 2 & & 0.70 & & 0.03 \\
\hline & 3 & & & 1.39 & 0.11 \\
\hline & 4 & & 0.57 & 1.29 & 0.13 \\
\hline \multirow[t]{4}{*}{$22: 5 n-6$} & 1 & 1.12 & & & 0.07 \\
\hline & 2 & & -0.16 & & 0.00 \\
\hline & 3 & & & -0.77 & 0.04 \\
\hline & 4 & 0.92 & & -0.51 & 0.09 \\
\hline \multirow[t]{4}{*}{$22: 5 n-3$} & 1 & $2.57^{*}$ & & & 0.29 \\
\hline & 2 & & -0.48 & & 0.01 \\
\hline & 3 & & & -0.30 & 0.01 \\
\hline & 4 & $2.48^{*}$ & 0.39 & & 0.30 \\
\hline \multirow[t]{4}{*}{$22: 6 n-3$} & 1 & $3.28 * *$ & & & 0.40 \\
\hline & 2 & & -0.70 & & 0.03 \\
\hline & 3 & & & 0.06 & 0.00 \\
\hline & 4 & $3.40^{* *}$ & & 0.95 & 0.44 \\
\hline \multirow[t]{4}{*}{$\sum$ PUFA } & 1 & $3.84 * *$ & & & 0.48 \\
\hline & 2 & & -0.95 & & 0.05 \\
\hline & 3 & & & -0.06 & 0.00 \\
\hline & 4 & $3.93^{* *}$ & & 0.92 & 0.51 \\
\hline \multirow[t]{4}{*}{$\sum \mathrm{FA}$} & 1 & $4.36^{* *}$ & & & 0.54 \\
\hline & 2 & & -1.12 & & 0.07 \\
\hline & 3 & & & -0.11 & 0.00 \\
\hline & 4 & $4.47^{* *}$ & & 0.99 & 0.57 \\
\hline
\end{tabular}

For each variable, the $t$ ratios are included. Models 1-4 evaluated the following: 1: turbidity; 2: salinity; 3: water temperature; 4: more explanatory pair of environmental parameters. ${ }^{*} P<0.05, * * P<0.01,{ }^{\text {a }}$ marginally significant $(P=0.05)$

Generally, the seasonal trend in the content of individual AA in E. siliqua exhibited two peaks (June and February) and two minimums (April and December) (Table 2). Though not statistically significant for all AAs, an increase in AA content is observable between April and June ( $\sum$ EAA, $\sum$ NEAA, $\sum A A$; Figs. 2D, 3C, D), which is coincident with the spawning period of E. siliqua (Gaspar and Monteiro 1998; Darriba et al. 2005b). Yet, given the release of gametes and therefore the loss of protein-bound amino acids contained in eggs and sperm (Bressan and Marin 1985; Tarnowska et al. 2009; Hamdani and SoltaniMazouni 2011), a decrease in $\sum A A$ content would be expected to occur. In fact, marine bivalve eggs are mainly composed of proteins, with these compounds accounting for 32.9-74.0\% of their dry weight (Bayne et al. 1978; Pieters et al. 1980; Lee and Heffernan 1991; Massapina et al. 1999; Park et al. 2003). Moreover, pod razor clam eggs are relatively large (i.e., $99.3 \pm 3.2 \mu \mathrm{m}$ in diameter; da Costa 2009) and the egg mass in adult bivalve females was shown to reach $26.8-38.9 \%$ of the individuals total dry weight (Park et al. 2003; Park and Choi 2004). Nonetheless, similar trends in protein content were recorded for other bivalve species. In the study of Davis and Wilson (1983), an increase in protein content of Nucula turgida was observed immediately after the main spawning period. In Spisula solida, an increase in the protein content was witnessed in the period immediately after the onset of spawning (Joaquim et al. 2008). Last, in the study of Yang et al. (2011), protein content of Fulvia mutica increased during the first spawning period. Neither study attempted to explain such results. An increase in the protein content in adductor muscles was shown to be associated with increasing water temperature and food availability (Taylor and Venn 1979). In fact, in the three mentioned studies and 
presumably in the present one, spawning occurred during a period of increasing or high water temperature (Fig. 5C). Additionally, a high amount of food was available at Caldeira de Tróia in the Spring of 2010 (Fig. 5A). Interestingly, in the present study, turbidity and water temperature were found not responsible for the measurable AA content fluctuations in E. siliqua (Fig. 5; Table 4). On the other hand, as mentioned above, spawning is known to be responsible for AA loss and as such is bound to have impacted AA content. It is possible that the simultaneous influence of these three factors has obscured their individual roles in AA dynamics. It then appears that the combined effect of increasing temperature and high food availability masked the loss of AAs resulting from gamete release. Still, a comparatively greater increase was registered for glutamic acid and leucine (April-June, Table 2). Such result may be indicative of an important role of these AAs at this stage of the reproductive cycle. Evidence of AAs involvement in reproduction exists for a variety of terrestrial and marine organisms (e.g., Kasschau and McCommas 1982; O'Brien et al. 2005; Wu et al. 2008) hence opening the possibility of such compounds holding a similar role in bivalve mollusks. Given the coincidence between gamete release and the increase in the contents of glutamic acid and leucine, it appears that these two AAs are involved in gonad maintenance, possibly as part of a post-spawning recovery mechanism. Free AAs (FAAs) are known osmolytes playing an important role in the osmoregulation of bivalves. Under hypoosmotic stress, the intracellular concentration of FAAs decreases as a result of release, while under hyperosmotic stress, the intracellular concentration of FAAs increases as a result of uptake (Zurburg and De Zwaan 1981; Matsushima and Hayashi 1988). The roles of alanine and glycine in osmoregulation, for example, have been studied considerably (Baginski and Pierce 1977; Ellis et al. 1985). Yet, none of the observed variations in the seasonal content of AAs appears to have occurred as a result of salinity changes (Fig. 5B).

Fatty acid profile and seasonality

The main SFAs were 16:0 and 18:0, while the main MUFAs were 18:1n-9 and 18:1n-7. PUFA fraction was dominated by $n-3$ FAs, mainly 22:6n-3, 16:3n-3 and 20:5n-3 surpassing 16:3n-3 in April and June. Overall, the main FAs were 16:0 and 22:6n-3 during the entire studied period. Similar results were obtained for several other marine bivalves, including S. patula (Lewin et al. 1979; Beninger and Stephan 1985; Teshima et al. 1990; Wenne and Polak 1992; Pazos et al. 1996). EFA content ranged between 34.02 and $87.94 \mathrm{mg} \mathrm{g}^{-1} \mathrm{DW}$ in E. siliqua. In their study, Wenne and Styczyńska-Jurewicz (1987) reviewed the lipid content of 31 bivalve species. Values varied between approx. 20.00-345.00 $\mathrm{mg} \mathrm{g}^{-1} \mathrm{DW}$, therefore placing $E$. siliqua in the lower range of lipid content among bivalves. Interestingly, lipid content found for $S$. patula (i.e., 400.00-430.00 $\mathrm{mg} \mathrm{g}^{-1}$ DW; Lewin et al. 1979) far exceeds that of $E$. siliqua while also exceeding the values reviewed by Wenne and Styczyńska-Jurewicz (1987).

Generally, the seasonal trend in the content of individual FAs in E. siliqua exhibited one peak in April and a minimum either in October or December (Table 3). Differently from what occurs with $\sum \mathrm{AA}$ content, the seasonal variation observed in $\sum F A$ content follows the trend described in most studies with FA levels increasing with gametogenesis and subsequently dropping with spawning (Fig. 4F; Pieters et al. 1980; Bressan and Marin 1985; Tarnowska et al. 2009). EFA content is highest in April, when sea water temperature is relatively low (Fig. 5C). A decrease in $\sum F A$ content is observed between April and June, when greater food availability, resulting from Spring blooms (Fig. 5A), should have allowed for the accumulation of FAs (Bressan and Marin 1985; Jarzebski et al. 1986). This decrease takes place during the spawning season (Gaspar and Monteiro 1998; Darriba et al. 2005b) and should therefore be related to the loss of FAs contained in gametes (Taylor and Venn 1979; Ojea et al. 2004; Hamdani and Soltani-Mazouni 2011). In fact, lipids are the second main component of marine bivalve eggs, accounting for 11.4-38.0\% of their dry weight (Bayne et al. 1978; Pieters et al. 1980; Lee and Heffernan 1991; Massapina et al. 1999; Park et al. 2003) or even 47.5 \% DW (Davis and Wilson 1983). In the present study, biochemical analysis was performed on whole-body tissue. Gonad-specific biochemical fluctuations, however, become diluted in wholebody dynamics (Ojea et al. 2004). Consequently, loss of gonadal FAs may have been masked by FA fluctuations related to other tissues (e.g., adductor muscle, gills). Between August and October, period comprising the reproductively inactive period (Gaspar and Monteiro 1998; Darriba et al. 2005b), $\sum$ FA levels remained low and relatively stable. During the first stages of gametogenesis, lipids accumulate in the digestive gland. Later, as gonads grow, lipids are accumulated in gametes (Barber and Blake 1981). Through comparison of $\sum F A$ values in April and December, it is plausible that an increase in FA content occurs throughout gametogenesis (Gaspar and Monteiro 1998; Darriba et al. 2005b). Thus, the main seasonal changes in $\sum F A$ content of E. siliqua appear strongly connected with the reproductive cycle.

The content of some FAs did not exhibit the general seasonal trend followed by the rest. Between April and June (spawning period) a comparatively greater decrease in $14: 0,16: 0,16: 1 n-7,18: 1 n-7,18: 1 n-9,18: 3 n-3,18: 4 n-3$, 20:1n-9 and 20:2n-6 was observed. Similar results were obtained in the work of Beninger and Stephan (1985) while 
analyzing the seasonal variations in whole-body FA composition of $V$. decussata and Venerupis philippinarum and in the works of Besnard et al. (1989) and Ojea et al. (2004) while analyzing the FA composition of gonads from Pecten maximus and $V$. decussata, respectively. Moreover, the FAs 14:0, 16:0, 16:1n-7, 18:1n-7 and 18:1n-9 were found to be among the major FAs in unfertilized eggs of $V$. decussata (Ojea et al. 2004). Such result is indicative of the significance of these FAs in bivalve eggs, possibly possessing a crucial role in embryonic development. During gametogenesis (i.e., between December and April) the contents of 14:0, 16:0, 16:1n-7, 18:1n-9, 18:3n-3, 18:4n-3, 20:1n-9, 20:2n-6 and 20:5n-3 increase considerably more than other FAs. Similar results were obtained for Mytilus edulis females in the months preceding spawning (Kluytmans et al. 1985). Also, Besnard et al. (1989) found maximal concentrations of $18: 3 n-3,18: 4 n-3$ and $20: 5 n-3$ to be related to oocyte maturation in $P$. maximus. These results suggest that such FAs are involved in oocyte maturation, especially $18: 3 n-3,18: 4 n-3,20: 1 n-9$ and 20:2n-6 which are not actively accumulated in unfertilized eggs (Ojea et al. 2004). The FA 22:4n-6, on the other hand, increases with spawning when practically all other FAs decrease, and thus, it is plausible to assume that this FA possesses a chief role in gonad maintenance, possibly as part of a post-spawning recovery mechanism (see discussion about AA involvement in gonad maintenance in the previous sub-section). It is also noteworthy that none of the observed variations in the seasonal content of FAs occurred as a result of turbidity, salinity or temperature changes (Fig. 5; Table 5).

The results on the AA and FA composition of $E$. siliqua presented herein provide a baseline for future research. Also, the one-year period of study provides insight on the seasonal fluctuations of these compounds. By performing similar studies in subsequent years, for example, it will be possible for ecologists to register the interannual differences in biochemical composition and how environmental change will affect it. On a larger scale, comparison of the presented data with that of populations from other regions may allow for the direct analysis of the impact of environmental forcing. On a more economical point of view, the presented data on biochemical condition may be used by E. siliqua-directed fisheries on the selection of the harvesting period in order to maximize the nutritional quality of the catch. Lastly, aquaculture systems may benefit from the presented data. In their work, da Costa et al. (2010) pointed out that improvement of postlarval survival could lead to a large-scale development of E. siliqua aquaculture. Condition of bivalve adults has been shown to influence nutrient levels of eggs (Bayne et al. 1978), number of larvae produced and larvae growth rate (Helm et al.
1973). Complementarily, larvae viability was shown to be significantly correlated with their lipid level (Helm et al. 1973). It is possible that through the usage of a feed supplement enriched with the above highlighted AAs and FAs (potentially involved in different stages of the reproductive process), both condition of adults and survival of spats could be improved in E. siliqua and other bivalve species.

\section{Conclusions}

Here, we have shown that in E. siliqua, the AA and FA seasonal fluctuations did not follow the same general trend. FAs increased with gametogenesis and decreased with spawning while AA content increased throughout spawning. In AA dynamics, the combined effect of increasing temperature and high food availability seem to have masked the loss of AAs resulting from gamete release. Still, involvement of AAs in post-spawning gonad recovery may occur. Salinity did not have an effect on the AA composition. On the other hand, certain FAs appear to be deeply involved in different stages of the reproductive process (i.e., embryonic development, gametogenesis, post-spawning gonad recovery). None of the three environmental parameters measured: turbidity, salinity and water temperature had an effect on FA composition.

Acknowledgments The authors would like to thank the anonymous reviewer for the constructive comments and valuable suggestions which helped improve this manuscript. The Portuguese Foundation for Science and Technology (FCT) supported this study through a Senior Research Position (Ciencia 2007) to R.R.

\section{Appendix}

See Tables 2, 3, 4, 5

\section{References}

Allen K (1961) Amino acids in the Mollusca. Am Zool 1:253-261 AOAC (2005) Official methods of analysis of the Association of Official Analytical Chemists International. Gaithersburg, MD, AOAC

Baginski RM, Pierce SK (1977) The time course of intracellular free amino acid accumulation in tissues of Modiolus demissus during high salinity adaptations. Comp Biochem Physiol A 57:407-412

Baptista M, Lopes VM, Pimentel MS, Bandarra N, Marques A, Rosa $\mathrm{R}$ (2012) Temporal fatty acid dynamics of the octocoral Veretillum cynomorium. Comp Biochem Physiol B 161:178-187

Barber BJ, Blake NJ (1981) Energy storage and utilization in relation to gametogenesis in Argopecten irradians concentricus (say). J Exp Mar Biol Ecol 52:121-134 
Barbour Wood SL, Krause J, Richard A, Kowalewski M, Wehmiller J, Simões MG (2006) Aspartic acid racemization dating of Holocene brachiopods and bivalves from the southern Brazilian shelf, South Atlantic. Quat Res 66:323-331

Bayne BL, Holland DL, Moore MN, Lowe DM, Widdows J (1978) Further studies on the effects of stress in the adult on the eggs of Mytilus edulis. J Mar Biol Assoc U K 58:825-841

Beninger PG, Stephan G (1985) Seasonal variations in the fatty acids of the triacylglycerols and phospholipids of two populations of adult clam (Tapes decussatus L. and T. Philippinarum) reared in a common habitat. Comp Biochem Physiol B 81:591-601

Besnard J-Y, Lubet P, Nouvelot A (1989) Seasonal variations of the fatty acid content of the neutral lipids and phospholipids in the female gonad of Pecten maximus L. Comp Biochem Physiol B 93:21-26

Beukema JJ, De Bruin W (1977) Seasonal changes in dry weight and chemical composition of the soft parts of the tellinid bivalve Macoma balthica in the Dutch Wadden Sea. Neth J Sea Res 11:42-55

Breen M, Howell T, Copland P (2011) A report on electrical fishing for razor clams (Ensis sp.) And its likely effects on the marine environment. Marine Scotland Science Report 03/11, Scotland

Bressan M, Marin MG (1985) Seasonal variations in biochemical composition and condition index of cultured mussels (Mytilus galloprovincialis Lmk) in the Lagoon of Venice (North Adriatic). Aquaculture 48:13-21

Cohen Z, Von Shak A, Richmond A (1988) Effect of environmental conditions on fatty acid composition of the red algae Porphyridium cruentum: correlation to growth rate. J Phycol 24:328-332

da Costa F (2009) Optimización del cultivo en criadero de navaja (Ensis arcuatus Jeffreys, 1865), longueirón (E. siliqua Linneo, 1758) y longueirón vello (Solen marginatus Pennánt, 1777): composición bioquímica y de ácidos grasos en los desarrollos larvarios. Dissertation, University of Santiago de Compostela

da Costa F, Martínez-Patiño D, Ojea J, Nóvoa S (2010) Larval rearing and spat production of the razor clam Ensis siliqua (Bivalvia: Pharidae). J Shellfish Res 29:347-351

Darriba S, San Juan F, Guerra A (2005a) Energy storage and utilization in relation to the reproductive cycle in the razor clam Ensis arcuatus (Jeffreys, 1865). ICES J Mar Sci 62:886-896

Darriba S, San Juan F, Guerra A (2005b) Gametogenic cycle of Ensis Siliqua (Linnaeus, 1758) in the Ría De Corcubión, Northwestern Spain. J Molluscan Stud 71:47-51

Davis JP, Wilson JG (1983) Seasonal changes in tissue weight and biochemical composition of the bivalve Nucula turgida in Dublin Bay with reference to gametogenesis. Neth J Sea Res 17:84-95

Dussart GBJ (1983) The amino acid composition of freshwater mollusc shells in relation to phylogeny and environment. J Molluscan Stud 49:213-223

Ellis LL, Burcham JM, Paynter KT, Bishop SH (1985) Amino acid metabolism in euryhaline bivalves: regulation of glycine accumulation in ribbed mussel gills. J Exp Zool 233:347-358

Fatima M (1996) Growth indices, nutritive value and chemical significance of the green mussel. Dissertation, University of Karachi

Fernández-Tajes J, Gaspar M, Martínez-Patiño D, McDonough N, Roberts D, González-Tizón A, Martínez-Lage A, Méndez J (2007) Genetic variation of the razor clam Ensis siliqua (Jeffreys, 1875) along the European coast based on random amplified polymorphic DNA markers. Aquac Res 38:1205-1212

Fernández-Tajes J, Arias-Pérez A, Fernández-Moreno M, Méndez J (2012) Sharp decrease of genetic variation in two Spanish localities of razor clam Ensis siliqua: natural fluctuation or Prestige oil spill effects? Ecotoxicol 21:225-233
Gabbott PA (1983) Developmental and seasonal metabolic activities in marine molluscs. Mollusca, Vol 2. Academic Press, New York

Gaspar MB, Monteiro CC (1998) Reproductive cycles of the razor clam Ensis siliqua and the clam Venus striatula off Vilamoura, Southern Portugal. J Mar Biol Assoc U K 78:1247-1258

Gaspar MB, Castro M, Monteiro CC (1999) Effect of tooth spacing and mesh size on the catch of the Portuguese clam and razor clam dredge. ICES J Mar Sci 56:103-110

Goodfriend GA, Flessa KW, Hare PE (1997) Variation in amino acid epimerization rates and amino acid composition among shell layers in the bivalve Chione from the Gulf of California. Geochim Cosmochim Acta 61:1487-1493

Hamdani A, Soltani-Mazouni N (2011) Changes in biochemical composition of the gonads of Donax trunculus L. (Mollusca, Bivalvia) from the Gulf of Annaba (Algeria) in relation to reproductive events and pollution. Jordan J Biol Sci 4:149-156

Hauton C, Atkinson RJA, Moore PG (2003) The impact of hydraulic blade dredging on a benthic megafaunal community in the Clyde Sea area, Scotland. J Sea Res 50:45-56

Hayward A, Gillooly J (2011) The cost of sex: quantifying energetic investment in gamete production by males and females. PLoS ONE 6(1):e16557

Helm MM, Holland DL, Stephenson RR (1973) The effect of supplementary algal feeding of a hatchery breeding stock of Ostrea Edulis L. on larval vigour. J Mar Biol Assoc U K 53:673-684

Henderson JW, Ricker RD, Bidlingmeyer BA, Woodward C (2000) Rapid, accurate, sensitive and reproducible analysis of amino acids. Agilent Publication Number 5980-1193ENAgilent Technologies, Palo Alto, CA

Holland DL (1978) Lipid reserves and energy metabolism in the larvae of benthic marine invertebrates. In: Sargent DC, Malins JR (eds) Biochemical and biophysical perspectives in Marine Biology. Academic Press, London, pp 85-123

IMAR (2008-2011) Relatorio do Programa de Monitorizacao Ambiental do projecto da Marina e novo Cais dos "ferries" do Troiaresort. Lisboa, Portugal, p 303

Jarzebski A, Wenne R, Habermehl G (1986) Anatomical distribution of lipids and sterols in Macoma balthica (L.). Comp Biochem Physiol B 85:135-137

Joaquim S, Matias D, Lopes B, Arnold WS, Gaspar MB (2008) The reproductive cycle of white clam Spisula solida (L.) (Mollusca: Bivalvia): implications for aquaculture and wild stock management. Aquaculture 281:43-48

Kasschau MR, McCommas SA (1982) Glycine concentration as a biochemical indicator of sex and maturation in the sea anemone Bunodosoma cavernata. Comp Biochem Physiol A 72:595-597

Kluytmans JH, Boot JH, Oudejans RCHM, Zandee DI (1985) Fatty acid synthesis in relation to gametogenesis in the mussel Mytilus edulis L. Comp Biochem Physiol B 81:959-963

Knauer J, Southgate PC (1999) A review of the nutritional requirements of bivalves and the development of alternative and artificial diets for bivalve aquaculture. Rev Fish Sci 7:241-280

Kube S, Sokolowski A, Jansen JM, Schiedek D (2007) Seasonal variability of free amino acids in two marine bivalves, Macoma balthica and Mytilus spp., in relation to environmental and physiological factors. Comp Biochem Physiol A 147:1015-1027

Lebour MV (1938) Notes on the breeding of some lamellibranchs from Plymouth and their larvae. J Mar Biol Assoc UK 23:119-145

Lee RF, Heffernan PB (1991) Lipids and proteins in eggs of eastern oysters (Crassostrea virginica (Gmelin, 1791)) and northern quahogs (Mercenaria mercenaria (Linnaeus, 1758)). J Shellfish Res 10:203-206

Lewin J, C-h Chen, Hruby T (1979) Blooms of surf-zone diatoms along the coast of the Olympic Peninsula, Washington. X. Chemical composition of the surf diatom Chaetoceros armatum and its 
major herbivore, the Pacific razor clam Siliqua patula. Mar Biol $51: 259-265$

Linnaeus C (1758) Tomus I. Systema naturae per regna tria naturae, secundum classes, ordines, genera, species, cum characteribus, differentiis, synonymis, locis. Holmiae (Laurentii Salvii)

Massapina C, Joaquim S, Matias D, Devauchelle N (1999) Oocyte and embryo quality in Crassostrea gigas (Portuguese strain) during a spawning period in Algarve, South Portugal. Aquat Living Resour 12:327-333

Matsushima O, Hayashi YS (1988) Uptake and accumulation of amino acids in the brackish-water bivalve Corbicula japonica Prime during high salinity acclimation. J Exp Mar Biol Ecol 123:201-210

Monteiro C, Gaspar MB (1993) Bivalves do litoral oceânico algarvio: breve notícia sobre a situação dos principais bancos (julho 1993). Relat Técn Cien, vol 65. INIP, Lisboa 65:19

O'Brien DM, Boggs CL, Fogel ML (2005) The amino acids used in reproduction by butterflies: a comparative study of dietary sources using compound-specific stable isotope analysis. Physiol Biochem Zool 78:819-827

Ojea J, Pazos AJ, Martínez D, Novoa S, Sánchez JL, Abad M (2004) Seasonal variation in weight and biochemical composition of the tissues of Ruditapes decussatus in relation to the gametogenic cycle. Aquac 238:451-468

Özden Ö, Erkan N (2011) A preliminary study of amino acid and mineral profiles of important and estimable 21 seafood species. Br Food J 113:457-469

Park K-I, Choi K-S (2004) Application of enzyme-linked immunosorbent assay for studying of reproduction in the Manila clam Ruditapes philippinarum (Mollusca: Bivalvia): I Quantifying eggs. Aquaculture 241:667-687

Park K-I, Choi J-W, Choi K-S (2003) Quantification of reproductive output of the butter clam, Saxidomus purpuratus (Sowerby, 1852) using enzyme-linked immunosorbent assay (ELISA). Ocean Polar Res 25:249-256

Pazos AJ, Ruíz C, García-Martin O, Abad M, Sánchez JL (1996) Seasonal variations of the lipid content and fatty acid composition of Crassostrea gigas cultured in El Grove, Galicia, N.W Spain. Comp Biochem Physiol B 114:171-179

Pieters H, Kluitmans JH, Zandee DI, Cadée GC (1980) Tissue composition and reproduction of Mytilus edulis in relation to food availability. Neth J Sea Res 14:349-361

Rosa R, Nunes ML (2003) Seasonal changes in nucleic acids, amino acids and protein content in juvenile Norway lobster (Nephrops norvegicus). Mar Biol 143:565-572

Rosa R, Nunes L, Reis CS (2002) Seasonal changes in the biochemical composition of Octopus vulgaris Cuvier, 1797, from three areas of the Portuguese coast. Bull Mar Sci 71:739-751

Sidwell VD, Loomis AL, Grodner RM (1979) Geographic and monthly variation in composition of oysters, Crassostrea virginica. Mar Fish Rev 41:13-17

Sokolowski A, Wolowicz M, Hummel H (2003) Free amino acids in the clam Macoma balthica L. (Bivalvia, Mollusca) from brackish waters of the southern Baltic Sea. Comp Biochem Physiol A 134:579-592

Tarnowska K, Wołowicz M, Chenuil A, Feral J-P (2009) Comparative studies on the morphometry and physiology of European populations of the lagoon specialist Cerastoderma glaucum (Bivalvia). Oceanol 51:437-458

Taylor AG, Savage C (2006) Fatty acid composition of New Zealand green-lipped mussels, Perna canaliculus: implications for harvesting for n-3 extracts. Aquac 261:430-439

Taylor AC, Venn TJ (1979) Seasonal variation in weight and biochemical composition of the tissues of the queen scallop, Chlamys opercularis, from the Clyde Sea Area. J Mar Biol Assoc U K 59:605-621

Tebble N (1966) British bivalve seashells: a handbook for identification. British Museum, Edinburgh

Teshima S, Kanazawa A, Koshio S, Mukai H, Yamasaki S, Hirata H (1990) Fatty acid details for bivalves, Tapes philippinarum and Corbicula japonica, and marine types of algae, Nannochloropsis sp. and Chlorella sp. Mem Fac Fish Kagoshima Univ 39:137-149

Trytek RE, Allen WV (1980) Synthesis of essential amino acids by bacterial symbionts in the gills of the shipworm Bankia setacea (tryon). Comp Biochem Physiol A 67:419-427

Tuck ID, Bailey N, Harding M, Sangster G, Howell T, Graham N, Breen M (2000) The impact of water jet dredging for razor clams, Ensis spp., in a shallow sandy subtidal environment. J Sea Res 43:65-81

von Cosel R, Gofas S (2011) Ensis siliqua (Linnaeus, 1758). World register of marine species. http://www.marinespecies.org/aphia. php?p=taxdetails\&id=140735. Accessed 13 August 2011

Wenne R, Polak L (1992) Lipid storage strategy in the clam Macoma (Bivalvia). Proceedings of the 12th Baltic Marine Biologists Symposium 179-183

Wenne R, Styczyńska-Jurewicz E (1987) Gross biochemical composition of the bivalve Macoma balthica from the Gulf of Gdańsk (Southern Baltic). Mar Biol 96:73-78

Wootton EC, Dyrynda EA, Ratcliffe NA (2003) Bivalve immunity: comparisons between the marine mussel (Mytilus edulis), the edible cockle (Cerastoderma edule) and the razor-shell (Ensis siliqua). Fish Shellfish Immunol 15:195-210

Wu G, Bazer F, Davis T, Kim S, Li P, Marc Rhoads J, Carey Satterfield M, Smith S, Spencer T, Yin Y (2008) Arginine metabolism and nutrition in growth, health and disease. Amino Acids 37:153-168

Yang H-S, Kang D-H, Park H-S, Choi K-S (2011) Seasonal changes in reproduction and biochemical composition of the cockle, Fulvia mutica Reeve (1884), in Cheonsu Bay off the west coast of Korea. J Shellfish Res 30:95-101

Zurburg W, De Zwaan A (1981) The role of amino acids in anaerobiosis and osmoregulation in bivalves. $\mathrm{J}$ Exp Zool 215:315-325 